\title{
Quantile Treatment Effects in Difference in Differences Models under Dependence Restrictions and with only Two Time Periods*
}

\author{
Brantly Callaway ${ }^{\dagger} \quad$ Tong $\mathrm{Li}^{\ddagger} \quad$ Tatsushi Oka ${ }^{\S}$ \\ The first version: December 15, 2015 \\ This version: February 14, 2017
}

\begin{abstract}
This paper shows that the Conditional Quantile Treatment Effect on the Treated can be identified using a combination of (i) a conditional Distributional Difference in Differences assumption and (ii) an assumption on the conditional dependence between the change in untreated potential outcomes and the initial level of untreated potential outcomes for the treated group. The second assumption recovers the unknown dependence from the observed dependence for the untreated group. We also consider estimation and inference in the case where all of the covariates are discrete. We propose a uniform inference procedure based on the exchangeable bootstrap and show its validity. We conclude the paper by estimating the effect of state-level changes in the minimum wage on the distribution of earnings for subgroups defined by race, gender, and education.
\end{abstract}

Keywords: Quantile Treatment Effects, Copula, Panel Data

JEL Classification: C14, C21, C23, C50

${ }^{*}$ We thank an Editor and two anonymous referees for their constructive comments which have greatly improved the paper. We also thank Yi-Ting Chen, Le-Yu Chen, Jiti Gao, Wolfgang Hardle, Yu-Chin Hsu, Hidehiko Ichimura, Brett Inder, Kengo Kato, Jen-Che Liao, Daisuke Nagakura, Anastasios Panagiotelis and participants of seminars at Academia Sinica, Keio University, Tokyo University and Monash University. The last author gratefully acknowledges the financial support from Singapore Ministry of Education Academic Research Fund Tier 1 (FY2015-FRC3-003).

${ }^{\dagger}$ Department of Economics, Temple University (brantly.callaway@temple.edu)

${ }^{\ddagger}$ Department of Economics, Vanderbilt University (tong.li@vanderbilt.edu)

$\S$ Department of Economics, National University of Singapore (oka@nus.edu.sg) 


\section{Introduction}

Researchers and policy makers are interested in evaluating the effect of participating in a program or experiencing a treatment but this is not a trivial task due to self-selection. Gronau (1974) and Heckman (1974) study the issue of self-selection in the context of labor markets and Amemiya (1985) provides a comprehensive framework for this issue in his influential book Advanced Econometrics, which also includes his seminal paper Amemiya (1973) on the Tobit model. In the literature, the Average Treatment Effect (ATE) or Average Treatment Effect on the Treated (ATT) has received great attention. But there are cases where a researcher may be interested in studying the distributional effect of treatment. In fact, estimating distributional treatment effect parameters is becoming more common in applied work. Recently, distributional treatment effects have been estimated in the context of welfare reform (Bitler et al., 2006, 2008), conditional cash transfer programs in developing countries (Djebbari and Smith, 2008), head start (Bitler et al., 2014), and the effect of Job Corps (Eren and Ozbeklik, 2014). One thing that each of the above empirical papers have in common is that each uses experimental data. In practice, however, experimental data is not always available and research for estimating distributional treatment effects in observational studies is of importance.

The current paper considers identification and estimation of a particular distributional treatment effect parameter called the Conditional Quantile Treatment Effect on the Treated (CQTT) under a Difference in Differences (DID) setup when only two periods of panel or repeated cross sections data are available. For applied researchers, it is not uncommon to have exactly two periods of data. For example, $25 \%$ of the papers employing DID assumptions considered by Bertrand et al. (2004) used exactly two periods of data. To give some specific examples, the Current Population Survery (CPS) Merged Outgoing Rotation Groups contains a 2-period panel (see Madrian and Lefgren, 2000; Riddell and Song, 2011, for instance) and the Displaced Workers Survey contains data on current wages and wages before displacement (see Farber, 1997, for example).

For identifying the CQTT, we consider a distributional extension of the "parallel trends" assumption, which is commonly used under the mean DID framework to ensure that the path of potential outcomes without treatment are the same between the treated and untreated groups on average. We extend this mean DID assumption to allow for the distribution of the path to be the same for the treated and untreated groups. This Distributional DID assumption is not strong enough to point-identify the counterfactual distribution of outcomes for the treated group as well as the CQTT. This is because identifying the counterfactual distribution for the treated group hinges on (i) knowing the distribution of the change in untreated potential outcomes and (ii) knowing the dependence, or copula, between the change and initial level of untreated potential outcomes. The Distributional DID assumption handles the first identification challenge but not the second. The key innovation of the current paper is to introduce the Copula Invariance assumption, which replaces the unknown copula with the observed copula between the change and initial level of outcomes for the untreated group and handles the second identification challenge. Importantly, the Copula Invariance assumption allows for the marginal distribution of untreated outcomes in the period before treatment to differ for the treated and control groups. Moreover, this assumption only requires that the researcher has access to two periods of data. 
Given the point-identification result for the counterfactual distribution, we propose a two-step estimation procedure based on conditional empirical distributions. In the first step, we estimate conditional empirical distributions of observed outcomes for the treated and untreated groups separately. In the second step, the first-step estimates are used in the estimation of the distribution of conditional counterfactual outcomes. The CQTT is estimated by inverting the estimated counterfactual distribution of potential outcome for the treated group. The proposed estimator is shown to converge in distribution to a Gaussian process at the parametric rate through empirical process techniques, while the limiting process is not nuisance parameter free because of estimation error at the first step and our identification strategy. To obtain an accurate approximation for the limiting process in a finite sample, we consider the exchangeable bootstrap proposed by Præstgaard and Wellner (1993) and show its first order validity.

This paper contributes to a growing literature on estimating quantile treatment effects (QTE) with observational data. The conditional QTE can be analyzed under the quantile regression framework proposed by Koenker and Bassett (1978). See Koenker (2005) for a comprehensive review. Abadie et al. (2002) and Chernozhukov and Hansen (2005, 2006, 2013) consider the conditional QTE allowing for endogeneous regressors in quantile regression when instrumental variables are available. For unconditional QTE, Firpo (2007) proposes a propensity score weighting estimator under a selection on observables assumption and Abadie (2003) and Frölich and Melly (2013) consider the case where a researcher has access to an instrument that satisfies the exclusion restriction only after conditioning on some covariates. Also, a counterfactual distribution of an outcome under a counterfactual covariates distribution has been studied by Firpo et al. (2009), Rothe (2012) and Chernozhukov et al. (2013a) among others.

Athey and Imbens (2006) suggest the Changes in Changes (CIC) model as an alternative to the DID model and Melly and Santangelo (2015) extend it to the case where the identifying assumptions hold conditional on covariates. Chernozhukov et al. (2013b) consider identification of the conditional ATE and QTE for nonseparable panel data models under a time-homogeneity condition. D'Haultfoeuille et al. (2015) present identification of nonseparable models using repeated cross-sections. Other recent contributions to panel quantile regression include Abrevaya and Dahl (2008), Canay (2011), Kato et al. (2012), Rosen (2012), Galvao et al. (2013), Galvao and Kato (2014), Chernozhukov et al. (2015), Graham et al. (2015), Li and Oka (2015), Arellano and Bonhomme (2016), Chetverikov et al. (2016) and Khan et al. (2016) among others.

Our work is closely related to Fan and Yu (2012) and Callaway and Li $(2015)$ in that both works consider the identification of the QTT under a Distributional DID assumption. Fan and $\mathrm{Yu}$ (2012) consider the partial identification of the QTT by using a Frechet-Hoeffding bound for a copula, while Callaway and Li (2015) establish the point-identification of the QTT using at least three periods of panel data by replacing an unknown, unconditional copula in the last two periods with an observed copula in the first two periods. In this paper, we focus on estimating conditional QTEs, while it would be relatively straightforward to extend our analysis to accommodate the unconditional QTE. Conditioning on covariates makes the Distributional DID assumption more likely to hold in empirical applications. For example, it seems likely that the path of earnings in the absence of treatment depends on individual characteristics such as age and experience. If these covariates are distributed 
differently across the treated and untreated groups, then an unconditional DID approach will not be valid though a conditional DID approach will (Heckman et al., 1998; Abadie, 2005). Similarly, our Copula Invariance assumption is more plausible when it holds conditional on covariates. We focus on identifying and estimating the CQTT in the case where all covariates are discrete which allows for nonparametric estimation that does not suffer from the curse of dimensionality and is a relevant case for much applied research. The identification and estimation with discrete regressors have been considered in Chernozhukov et al. (2013b) for the conditional ATE and QTE and Graham et al. (2015) for quantile panel data model with random coefficients.

We use our method to investigate the effect of increases in the minimum wage on the distribution of earnings. Using individual level panel data from the Current Population Survey, we consider a group of five treated states that increased their minimum wage in 2007 relative to a control group of states that did not increase the minimum wage. Forming groups based on race, gender, and education, we are only able to reject the null of no effect at any quantile for three out of eight groups. For these groups, the effect of the minimum wage is concentrated in the lower part of the distribution and appears to result in lower earnings.

The paper is organized as follows. In Section 2, we provide identification results for the CQTT under the Distributional DID assumption and the Copula Invariance assumption when both hold after conditioning on some covariates. In Section 3, we discuss an estimation procedure and provide asymptotic results. We also introduce a resampling procedure and give a theoretical result on its consistency. Section 4 investigates the finite sample performance of our estimator using Monte Carlo simulations. In Section 5, we present an empirical application on the effect of increasing the minimum wage on the distribution of earnings. Section 6 concludes. All proofs are given in the Appendix.

\section{Identification}

This section considers the main identification results in the paper. We consider identification of the QTT when the identifying assumptions hold after conditioning on some observed covariates $X$. We also consider additional requirements for identification when only repeated cross sections are available. Through the paper, we use $\operatorname{supp}(V)$ and $\operatorname{supp}(V \mid W)$ to denote the support of $V$ and the support of $V$ conditional on $W$, respectively, for some random variables $V$ and $W$.

\subsection{Identification}

We consider a DID framework, in which all individuals in the sample receive no treatment in period $t-1$ while a fraction of individuals receive the treatment in period $t$. Let $D_{i t}$ be a treatment indicator that takes the value one if individual $i$ is treated in period $t$ and zero otherwise. For each individual $i$, there is a pair of potential outcomes $\left(Y_{i s}(0), Y_{i s}(1)\right)$ in period $s \in\{t-1, t\}$, where $Y_{i s}(0)$ and $Y_{i s}(1)$ denote potential outcomes in the untreated and treated state in period $s$, respectively. Every individual experiences either treated or untreated status but not both, and thus the pair of potential outcomes is not observable. 
We suppose that researchers can access panel data, which consist of outcomes, covariates and treatment statuses for each individual over some periods including both before and after the implementation of the program of interest. We consider the case with two periods of panel data and so that the data consists of observations $\left\{\left(Y_{i, t-1}, Y_{i t}, X_{i}, D_{i t}\right)\right\}_{i=1}^{n}$ with $n$ denoting the sample size, where the observed outcomes are given by

$$
Y_{i, t-1}:=Y_{i, t-1}(0) \quad \text { and } \quad Y_{i t}:=\left(1-D_{i t}\right) Y_{i t}(0)+D_{i t} Y_{i t}(1)
$$

Also, $X_{i}$ denotes a vector of covariates, which may include all time-varying variables as well as time-invariant variables. Throughout the paper, we assume independent and identically distributed (i.i.d.) observations within treatment and control group as stated below.

Assumption A1 (Random sampling). The two-periods panel data consists of observations $\left\{\left(Y_{i, t-1}, Y_{i t}, X_{i}, D_{i t}\right)\right\}_{i=1}^{n}$ from the structure in $(1)$. The potential outcomes $\left(Y_{i, t-1}(0), Y_{i, t-1}(1)\right)$ and $\left(Y_{i t}(0), Y_{i t}(1)\right)$ are cross-sectionally i.i.d. conditional on treatment status $D_{i t}$.

This assumption allows for the possibility that the marginal or joint distributions of potential outcomes can be different between treatment and control groups. Under this conditional random sampling assumption, we use $F_{Y_{s}(0) \mid X, D_{t}}$ and $F_{Y_{s}(1) \mid X, D_{t}}$ to denote conditional distributions of the potential outcomes in period $s$ given covariates and treatment status and let $F_{Y_{s} \mid X, D_{t}}$ be the conditional distribution of the observed outcome $Y_{i s}$ in period $s$ given covariates and treatment status.

Our primary goal is to identify distributional features of treatment effects through conditional distributions of observed outcomes given covariates and $D_{i t}$. The issue of identification of treatment effects arises because the pair of potential outcomes is unobservable for each individual and thus marginal distributions of potential outcomes are not necessarily identified from data. For instance, the conditional distribution $F_{Y_{t}(1) \mid X, D_{t}=1}$ of a potential outcome $Y_{i t}(1)$ given $X_{i}$ and $D_{i t}=1$ can be identified by the observed distribution $F_{Y_{t} \mid X, D_{t}=1}$ of outcomes for the treated group. But the counterfactual distribution $F_{Y_{t}(0) \mid X, D_{t}=1}$ of untreated potential outcomes for the treated group cannot be identified generally from the sample. Thus, for identifying distributional features of treatment effects, we need to make additional restrictions.

As a measure of treatment effects, this paper considers the identification and estimation of the CQTT given $X_{i}=x$, which measures the quantile treatment effect within a subpopulation of individuals with treatment status $D_{i t}=1$ and common history $X_{i}=x$. Let $\mathcal{X}$ be the common support of $X_{i}$ for untreated and treated groups. The CQTT given $x \in \mathcal{X}$ at $\tau \in \mathcal{T} \subset(0,1)$ is defined as

$$
\Delta_{x}^{Q T T}(\tau):=F_{Y_{t}(1) \mid X=x, D_{t}=1}^{-1}(\tau)-F_{Y_{t}(0) \mid X=x, D_{t}=1}^{-1}(\tau),
$$

where $F_{Y_{t}(j) \mid X, D_{t}}^{-1}$ is the quantile function of $Y_{i t}(j)$ conditional on $X_{i}$ and $D_{i t}$, given by

$$
F_{Y_{t}(j) \mid X, D_{t}}^{-1}(\tau):=\inf \left\{y \in \mathbb{R}: F_{Y_{t}(j) \mid X, D_{t}}(y) \geq \tau\right\}, \quad j=0,1 .
$$

As discussed in the preceding paragraph, we can identify the distribution $F_{Y_{t}(1) \mid X=x, D_{t}=1}$ from observables. For identifying the CQTT, it remains to establish the identification of the 
counterfactual distribution $F_{Y_{t}(0) \mid X=x, D_{t}=1}$. To this end, we need to make three additional restrictions.

The first condition restricts a time-difference of potential outcomes without treatment, denoted by $\Delta Y_{i t}(0):=Y_{i t}(0)-Y_{i, t-1}(0)$, is independent of the treatment status $D_{i t}$ conditional on $X_{i}$.

Assumption A2. (Distributional Difference in Differences)

$$
\operatorname{Pr}\left\{\Delta Y_{i t}(0) \leq \Delta y \mid X_{i}, D_{i t}=1\right\}=\operatorname{Pr}\left\{\Delta Y_{i t}(0) \leq \Delta y \mid X_{i}, D_{i t}=0\right\},
$$

for all $\Delta y \in \operatorname{supp}\left(\Delta Y_{i t}(0) \mid X_{i}\right)$.

This assumption ensures that potential outcomes without treatment are comparable between treatment and control groups after taking a time-difference, conditional on covariates. An analogous condition employed under the mean DID framework is the "parallel trends" assumption:

$$
E\left[\Delta Y_{i t}(0) \mid X_{i}, D_{i t}=1\right]=E\left[\Delta Y_{i t}(0) \mid X_{i}, D_{i t}=0\right],
$$

which is necessary for identifying the ATT (see Heckman et al., 1998; Abadie, 2005). The distributional restriction in Assumption A2 replaces this standard mean restriction. If multiple pre-treatment periods in sample are available, then this assumption can be tested under a strict stationary assumption.

For the treated group, we can identify (i) the distribution $F_{Y_{t-1}(0) \mid X, D_{t}=1}$ of untreated potential outcomes in period $t-1$ from observed outcomes and (ii) the distribution $F_{\Delta Y_{t}(0) \mid X, D_{t}=1}$ of the change in untreated potential outcomes through Assumption A2 (the distributional DID). When these two distributions are identified, the average untreated potential outcome (and hence, the ATT) is identified. Without imposing an additional assumption, however, the CQTT is not point-identified, while the CQTT can be partially identified along the line of Fan and $\mathrm{Yu}(2012)$. This is because many possible distributions of untreated potential outcomes in period $t$ are observationally equivalent. For example, the distribution $F_{Y_{t}(0) \mid X, D_{t}=1}$ of untreated potential outcomes in period $t$ will be highly unequal if the change in untreated potential outcomes and the initial untreated outcome are strongly positively dependent. On the other hand, the distribution of untreated potential outcomes in period $t$ will be less unequal if the change and initial level of untreated potential outcomes are independent or negatively dependent.

The next condition imposes a restriction on the joint distribution $F_{\Delta Y_{t}(0), Y_{t-1}(0) \mid X, D_{t}}$ of $\Delta Y_{i t}(0)$ and $Y_{i, t-1}(0)$ conditional on $X_{i}$ and $D_{i t}$ through the copula $C_{\Delta Y_{t}(0), Y_{t-1}(0) \mid X, D_{t}}$ of $\Delta Y_{i t}(0)$ and $Y_{i, t-1}(0)$ conditional on $X_{i}$ and $D_{i t}$. By Sklar's theorem, we have

$$
F_{\Delta Y_{t}(0), Y_{t-1}(0) \mid X, D_{t}}(\Delta y, y)=C_{\Delta Y_{t}(0), Y_{t-1}(0) \mid X, D_{t}}\left(F_{\Delta Y_{t}(0) \mid X, D_{t}}(\Delta y), F_{Y_{t-1}(0) \mid X, D_{t}}(y)\right),
$$

for $(\Delta y, y) \in \operatorname{supp}\left(\Delta Y_{i t}(0), Y_{i, t-1}(0) \mid X_{i}, D_{i t}\right)$. The following condition requires an invariance of the conditional copula with respect to the treatment status $D_{i t}$ conditional on $X_{i}$.

Assumption A3 (Copula Invariance). For each $x \in \mathcal{X}$ and for all $(u, v) \in[0,1]^{2}$,

$$
C_{\Delta Y_{t}(0), Y_{t-1}(0) \mid X=x, D_{t}=1}(u, v)=C_{\Delta Y_{t}(0), Y_{t-1}(0) \mid X=x, D_{t}=0}(u, v) .
$$


Given a realized value of some random variable, the marginal distribution evaluated at this value can be interpreted as a ranking normalized to the unit interval. The conditional copula function captures some rank dependency between two variables $\Delta Y_{i t}(0)$ and $Y_{i, t-1}(0)$ conditional on $X_{i}$ and $D_{i t}$ and Assumption A3 requires that the dependency of ranking of these random variables $\Delta Y_{i t}(0)$ and $Y_{i, t-1}(0)$ are the same for the treated and control groups. As in Assumption A1, however, this assumption does not rule out the possibility that the joint distribution of $\Delta Y_{i t}(0)$ and $Y_{i, t-1}(0)$ conditional on $X_{i}$ and $D_{i t}$ varies between the treatment and control group. Likewise, Assumption A3 does not imply Assumption A2 because A3 restricts only the copula, not the marginal distribution of the change in untreated potential outcomes over time.

The Copula Invariance assumption recovers the missing dependence required to uniquely identify the counterfactual distribution of untreated potential outcomes for the treated group. It does so by replacing the unknown copula for the treated group with the known copula from the untreated group. Intuitively, if, for example, we observe that observations in the control group at the top of the distribution of initial outcomes tend to experience the largest increases in outcomes over time, the Copula Invariance assumption implies that this would also occur for the treated group. The Distributional DID assumption implies that the distribution of the change in outcomes is the same for the two groups. But the initial distribution of outcomes can be different for the two groups.

The Distributional DID assumption and the Copula Invariance assumption are not directly testable. However, in the spirit of placebo testing in DID models, they both can be tested using periods before the treated group becomes treated. One idea would be to directly test each assumption in these earlier periods. Another, simpler idea would be to implement our procedure in the earlier periods and test that $\Delta_{x}^{Q T T}(\tau)=0$ for all $\tau \in \mathcal{T}$.

As an additional identifying restriction, we assume continuity conditions on distributions of potential outcomes and its time-difference as below.

Assumption $\mathbf{A} 4$ (Continuous distributions). Each random variable of $\Delta Y_{i t}(0)$ and $Y_{i, t-1}(0)$ has a continuous distribution conditional on $X_{i}$ and $D_{i t}$ and a random variable $Y_{i t}(1)$ also has a continuous distribution conditional on $X_{i}$ and $D_{i t}=1$. Each distribution has a compact support with densities uniformly bounded away from 0 and $\infty$ over the support.

The continuity of marginal distributions conditional on treatment status guarantees that conditional copulas in Assumption A3 are unique and facilitates the identification analysis. More precisely, we obtain identification by employing the Rosenblatt transform, which is the distribution transform studied by Rosenblatt (1952). Also, Assumption A4 imposes a compact support assumption as in Athey and Imbens (2006) in order to avoid technical difficulties in the rest of analysis, while this condition is not used for our identification analysis and can be replaced by other conditions for the rest of the results.

Given the random sample in Assumption A1, the additional conditions in Assumption A2-A4 deliver the point-identification of the counterfactual distribution as in the following theorem. 
Theorem 1. Suppose that Assumption A1-A4 hold. Then,

$F_{Y_{t}(0) \mid X=x, D_{t}=1}(y)=\operatorname{Pr}\left\{\Delta Y_{i t}+F_{Y_{t-1} \mid X=x, D_{t}=1}^{-1} \circ F_{Y_{t-1} \mid X=x, D_{t}=0}\left(Y_{i, t-1}\right) \leq y \mid X_{i}=x, D_{i t}=0\right\}$,

for all $x \in \mathcal{X}$ and $y \in \operatorname{supp}\left(Y_{i t}(0) \mid X_{i}=x, D_{i t}=1\right)$.

The above theorem shows that the counterfactual distribution of interest can be identified from observed outcomes of untreated individuals. This implies that that treated and untreated groups must be similar in the distributional sense of not only marginal distribution but also some dependency over periods, and thus Assumption A2 and A3 play a crucial role for the identification as shown in its proof. An immediate consequence is the identification of the conditional QTT since the other distribution $F_{Y_{t}(1) \mid X=x, D_{t}=1}$ is identified by the distribution $F_{Y_{t} \mid X=x, D_{t}=1}$ of observed outcomes.

Another implication of Theorem 1 is that unconditional QTTs are identified using our approach. One can simply average over the covariates in the conditional counterfactual distribution in Theorem 1 to obtain an unconditional counterfactual distribution and then invert it to obtain the unconditional quantiles. Thus, our method can be comparable to the results that obtain unconditional QTTs; however, for the rest of the paper we focus only on the CQTT.

As an extension of the result above, we establish the identification of the counterfactual distribution when the available data consists of two-periods of repeated cross sections, rather than panel data. In the following corollary, we only consider the case with time invariant covariates, following the DID literature with repeated cross sections (Abadie, 2005; Melly and Santangelo, 2015). Even when the data generating process satisfies Assumption A1A4, the change in outcome over time, $Y_{i t}-Y_{i, t-1}$, is unobserved because each individual in sample is observed only in one period. To deal with the identification issue due to the data, we consider a restriction of the conditional rank invariance, which enables us to recover individual outcome in period $t$ by using the rank of outcome in period $t-1$ as formalized by the following corollary.

Corollary 1. Consider the repeated cross sections $\left\{\left(Y_{i s}, X_{i}, D_{i s}\right)\right\}_{i=1}^{m^{(s)}}$ in period $s \in\{t-1, t\}$ with $m^{(s)}$ being the sample size. Suppose that the data generating process for the repeated cross sections satisfy Assumption A1-A4 hold. If the conditional copula of $\left(Y_{i, t-1}(0), Y_{i, t}(0)\right)$ given $X_{i}=x$ and $D_{i t}=1$ satisfies the rank invariance: for every $(u, v) \in[0,1]^{2}$,

$$
C_{Y_{t-1}(0), Y_{t}(0) \mid X=x, D_{t}=1}(u, v)=\min \{u, v\},
$$

then, for $y \in \operatorname{supp}\left(Y_{i t}(0) \mid X_{i}=x, D_{i t}=1\right)$,

$F_{Y_{t}(0) \mid X=x, D_{t}=1}(y)=\operatorname{Pr}\left\{\widetilde{\Delta Y}_{i t}+F_{Y_{t-1} \mid X=x, D_{t}=1}^{-1} \circ F_{Y_{t-1} \mid X=x, D_{t}=0}\left(Y_{i, t-1}\right) \leq y \mid X_{i}=x, D_{i t}=0\right\}$, where $\widetilde{\Delta Y}_{i t}:=F_{Y_{t} \mid X, D_{t}=0}^{-1} \circ F_{Y_{t-1} \mid X, D_{t}=0}\left(Y_{i, t-1}\right)-Y_{i, t-1}$.

The additional assumption of conditional rank invariance says that, for observations with the same observed covariates, individuals maintain their rank in the distribution of outcomes 
over time. This assumption is weaker than unconditional rank invariance as some individuals can change their rank in the distribution of earnings over time. It does not imply nor is implied by the Copula Invariance assumption, nor does it imply conditional rank invariance between $\Delta Y_{i t}(0)$ and $Y_{i t-1}(0)$.

\section{Estimation and Inference}

As in the previous section, the counterfactual distribution is identified by distributions of observed outcomes conditional on covariates and treatment status. In this section, we first explain an estimation procedure based on conditional empirical distributions and then provide asymptotic results for the proposed estimator using the functional delta method. We develop uniform inference results using techniques from the literature on empirical processes (see, for example, van der Vaart and Wellner, 1996). In this section, we consider the case where all covariates are discrete, which allows for nonparametric estimation that does not suffer from the curse of dimensionality. The estimation with discrete regressors is also considered in Chernozhukov et al. (2013b) and Graham et al. (2015).

\subsection{Estimation}

We estimate the conditional distribution $F_{Y_{s} \mid X=x, D_{t}=d}$ of observed outcome $Y_{i s}$ given covariates $X_{i}=x$ and treatment status $D_{i t}=d$ by using the corresponding empirical distribution. For $d \in\{0,1\}$, let $\delta_{i, x}^{(d)}:=1\left\{X_{i}=x, D_{i t}=d\right\}$ and $n_{x}^{(d)}=\sum_{i=1}^{n} \delta_{i, x}^{(d)}$. Then, the estimator of $F_{Y_{s} \mid X=x, D_{t}=d}$ is given by, for $s \in\{t-1, t\}$ and $d \in\{0,1\}$,

$$
\hat{F}_{Y_{s} \mid X=x, D_{t}=d}(y):=\frac{1}{n_{x}^{(d)}} \sum_{i=1}^{n} 1\left\{Y_{i s} \leq y\right\} \delta_{i, x}^{(d)} .
$$

We denote an estimator for $F_{Y_{t}(1) \mid X=x, D_{t}=1}(y)$ by $\hat{F}_{Y_{t}(1) \mid X=x, D_{t}=1}(y)$, which is given by the empirical distribution $\hat{F}_{Y_{t} \mid X=x, D_{t}=1}(y)$ because $Y_{i t}=Y_{i t}(1)$ if $D_{i t}=1$. For estimation of the counterfactual distribution provided in Theorem 1, we obtain estimated quantiles $\hat{F}_{Y_{t-1} \mid X=x, D_{t}=1}^{-1}$ from the empirical distribution $\hat{F}_{Y_{t-1} \mid X=x, D_{t}=1}$ and then set

$$
\hat{F}_{Y_{t}(0) \mid X=x, D_{t}=1}(y):=\frac{1}{n_{x}^{(0)}} \sum_{i=1}^{n} 1\left\{\Delta Y_{i t}+\hat{F}_{Y_{t-1} \mid X=x, D_{t}=1}^{-1} \circ \hat{F}_{Y_{t-1} \mid X=x, D_{t}=0}\left(Y_{i, t-1}\right) \leq y\right\} \delta_{i, x}^{(0)},
$$

for $y \in \mathbb{R}$. We use estimated distribution functions $\hat{F}_{Y_{t}(1) \mid X=x, D_{t}=1}$ and $\hat{F}_{Y_{t}(0) \mid X=x, D_{t}=1}$ to obtain quantiles for each distribution. Then, the CQTT estimator is given by

$$
\hat{\Delta}_{x}^{Q T T}(\tau):=\hat{F}_{Y_{t}(1) \mid X=x, D_{t}=1}^{-1}(\tau)-\hat{F}_{Y_{t}(0) \mid X=x, D_{t}=1}^{-1}(\tau),
$$

for $(\tau, x) \in \mathcal{T} \times \mathcal{X}$. 


\section{$3.2 \quad$ Asymptotic Results}

We provide a functional central limit theorem for the CQTT estimator over $\mathcal{T}$, where $\mathcal{T}$ is assumed to be a compact subset strictly within the unit interval. We begin with a preliminary result on weak convergence of empirical distributions, which facilitates the use of the functional delta method with Hadamard differentiable maps. In what follows, we denote by $\mathcal{Y}_{s \mid x, d}:=\operatorname{supp}\left(Y_{i s} \mid X_{i}=x, D_{i t}=d\right)$ and $\mathcal{Y}_{s \mid x, 1}(j):=\operatorname{supp}\left(Y_{i s}(j) \mid X_{i}=x, D_{i t}=1\right)$ for $s \in\{t-1, t\}, d \in\{0,1\}$ and $j \in\{0,1\}$.

For each $(s, d, x) \in\{t-1, t\} \times\{0,1\} \times \mathcal{X}$, define empirical processes as

$$
\hat{G}_{s, x}^{(d)}(y):=\sqrt{n}\left(\hat{F}_{Y_{s} \mid X=x, D_{t}=d}(y)-F_{Y_{s} \mid X=x, D_{t}=d}(y)\right), \quad y \in \mathcal{Y}_{s \mid x, d} .
$$

Let $\tilde{Y}_{i t}:=\Delta Y_{i t}+F_{Y_{t-1} \mid X=x, D_{t}=1}^{-1} \circ F_{Y_{t-1} \mid X=x, D_{t}=0}\left(Y_{i, t-1}\right)$ and we use $\tilde{F}_{Y_{t}(0) \mid X=x, D_{t}=1}(y)$ to denote the infeasible estimator for the counterfactual distribution based on observations $\left\{\tilde{Y}_{i t}\right\}$ with $X_{i}=x$ and $D_{i t}=1$ as in (2). Define its empirical process as

$$
\tilde{G}_{t, x}^{(0)}(y):=\sqrt{n}\left(\tilde{F}_{Y_{t}(0) \mid X=x, D_{t}=1}(y)-F_{Y_{t}(0) \mid X=x, D_{t}=1}(y)\right), \quad y \in \mathcal{Y}_{t \mid x, 1}(0) .
$$

We make an additional assumption.

Assumption A6. (a) A pair of random variables $\left(\Delta Y_{i t}, Y_{i, t-1}\right)$ is continuously distributed conditional on $X_{i}$ and $D_{i t}=0$ over a compact support with a distribution $F_{\Delta Y_{t}, Y_{t-1} \mid X, D_{t}=0}$ and a density $f_{\Delta Y_{t}, Y_{t-1} \mid X, D_{t}=0}$. A random variable $\Delta Y_{i t}$ is continuously distributed conditional on $Y_{i, t-1}, X_{i}$, and $D_{i t}=0$ with a uniformly continuous density $f_{\Delta Y_{t} \mid Y_{t-1}, X, D_{t}=0}$ over a compact support. (b) The sample sizes $n_{x}^{(0)}$ and $n_{x}^{(1)}$ go to $\infty$ as $n \rightarrow \infty$, while $r_{x}^{(j)}:=\lim _{n \rightarrow \infty}\left(n / n_{x}^{(j)}\right)^{1 / 2} \in(0, \infty)$ for $j=0,1$.

The following lemma provides a functional central limit theorem for the empirical processes above. We define $\mathbb{S}_{x}:=\ell^{\infty}\left(\mathcal{Y}_{t \mid x, 1}(0)\right) \times \ell^{\infty}\left(\mathcal{Y}_{t-1 \mid x, 0}\right) \times \ell^{\infty}\left(\mathcal{Y}_{t \mid x, 1}\right) \times \ell^{\infty}\left(\mathcal{Y}_{t-1 \mid x, 1}\right)$ for a fixed $x \in \mathcal{X}$, where $\ell^{\infty}(S)$ denotes the space of all uniformly bounded functions on some set $S$, equipped with supremum norm $\|\cdot\|_{\infty}$.

Lemma 1. Suppose that Assumption A1-A6 hold. Then, for each $x \in \mathcal{X}$,

$$
\left(\tilde{G}_{t, x}^{(0)}, \hat{G}_{t-1, x}^{(0)}, \hat{G}_{t, x}^{(1)}, \hat{G}_{t-1, x}^{(1)}\right) \rightsquigarrow\left(\mathbb{V}_{x}^{(0)}, \mathbb{W}_{x}^{(0)}, \mathbb{V}_{x}^{(1)}, \mathbb{W}_{x}^{(1)}\right)
$$

in the space $\mathbb{S}_{x}$. Here, $\left(\mathbb{V}_{x}^{(0)}, \mathbb{W}_{x}^{(0)}, \mathbb{V}_{x}^{(1)}, \mathbb{W}_{x}^{(1)}\right)$ is a tight Gaussian process with mean zero and covariance kernel $\operatorname{diag}\left\{\Sigma_{x}^{(0)}(\cdot, \cdot), \Sigma_{x}^{(1)}(\cdot, \cdot)\right\}$ defined on $\mathbb{S}_{x}$, where $\Sigma_{x}^{(j)}(\cdot, \cdot)$ is the $2 \times 2$ positive definite, covariance kernel of $\left(\mathbb{V}^{(j)}, \mathbb{W}^{(j)}\right)$ for $j=0,1$, given by, for $\left(y_{1}, y_{2}, y_{3}, y_{4}\right) \in \mathbb{S}_{x}$,

$$
\Sigma_{x}^{(0)}\left(y_{1}, y_{2}\right):=\operatorname{var}_{x}^{(0)}\left(I_{i t}^{(0)}\left(y_{1}, y_{2}\right)\right) \quad \text { and } \quad \Sigma_{x}^{(1)}\left(y_{3}, y_{4}\right):=\operatorname{var}_{x}^{(1)}\left(I_{i t}^{(1)}\left(y_{3}, y_{4}\right)\right)
$$

with $I_{i t}^{(0)}\left(y_{1}, y_{2}\right):=\left(1\left\{\tilde{Y}_{i t} \leq y_{1}\right\}, 1\left\{Y_{i, t-1} \leq y_{2}\right\}\right)^{\prime}, I_{i t}^{(1)}\left(y_{3}, y_{4}\right):=\left(1\left\{Y_{i t} \leq y_{3}\right\}, 1\left\{Y_{i, t-1} \leq y_{4}\right\}\right)^{\prime}$ and $\operatorname{var}_{x}^{(j)}$ being the covariance function conditional on $X_{i}=x$ and $D_{i t}=j$. 
Using the result in this lemma, we first obtain the joint limiting process for the estimator of the potential outcome distributions $\left(\hat{F}_{Y_{t}(0) \mid X=x, D_{t}=1}, \hat{F}_{Y_{t}(1) \mid X=x, D_{t}=1}\right)$. It is straightforward from the above result to obtain the limit process for the distribution $\hat{F}_{Y_{t}(1) \mid X=x, D_{t}=1}$, which is identified directly from data; whereas, the one for the counterfactual distribution $\hat{F}_{Y_{t}(0) \mid X=x, D_{t}=1}$ needs several steps. Since the estimator for the counterfactual distribution in (3) can be considered as a process indexed by functions depending on estimated distributions, we use recent results for empirical processes in van der Vaart and Wellner (2007) with some modifications in order to obtain the limiting process as formalized by the following proposition.

Proposition 1. Define $\hat{Z}_{x}^{(j)}(y):=\sqrt{n}\left(\hat{F}_{Y_{t}(j) \mid X=x, D_{t}=1}(y)-F_{Y_{t}(j) \mid X=x, D_{t}=1}(y)\right)$ for each $x \in \mathcal{X}$, $j=0,1$ and $y \in \mathcal{Y}_{t \mid x, 1}(j)$. Suppose that Assumption A1-A6 hold. Then,

$$
\left(\hat{Z}_{x}^{(0)}, \hat{Z}_{x}^{(1)}\right) \rightsquigarrow\left(\mathbb{Z}_{x}^{(0)}, \mathbb{Z}_{x}^{(1)}\right),
$$

in the metric space $\ell^{\infty}\left(\mathcal{Y}_{t \mid x, 1}(0)\right) \times \ell^{\infty}\left(\mathcal{Y}_{t \mid x, 1}(1)\right)$. Here, $\left(\mathbb{Z}_{x}^{(0)}, \mathbb{Z}_{x}^{(1)}\right)$ is a tight zero-mean Gaussian process with a.s. uniformly continuous paths on $\mathcal{Y}_{t \mid x, 1}(0) \times \mathcal{Y}_{t \mid x, 1}(1)$, given by

$$
\mathbb{Z}_{x}^{(0)}:=r_{x}^{(0)} \mathbb{V}_{x}^{(0)}+\kappa_{x}\left(\mathbb{W}_{x}^{(0)}, \mathbb{W}_{x}^{(1)}\right) \quad \text { and } \quad \mathbb{Z}_{x}^{(1)}=r_{x}^{(1)} \mathbb{V}_{x}^{(1)},
$$

where the map $\kappa_{x}: \ell^{\infty}\left(\mathcal{Y}_{t-1 \mid x, 0}\right) \times \ell^{\infty}\left(\mathcal{Y}_{t-1 \mid x, 1}\right) \mapsto \ell^{\infty}\left(\mathcal{Y}_{t \mid x, 1}(0)\right)$ is given by

$$
\kappa_{x}\left(W_{0}, W_{1}\right):=\int\left\{r_{x}^{(0)} W_{0}(v)-r_{x}^{(1)} W_{1} \circ F_{Y_{t-1} \mid X=x, D_{t}=1}^{-1} \circ F_{Y_{t-1} \mid X=x, D_{t}=0}(v)\right\} \omega_{x}(y, v) d v,
$$

for $\left(W_{0}, W_{1}\right) \in \ell^{\infty}\left(\mathcal{Y}_{t-1 \mid x, 0}\right) \times \ell^{\infty}\left(\mathcal{Y}_{t-1 \mid x, 1}\right)$ with

$$
\omega_{x}(y, v):=\frac{f_{\Delta Y_{t}, Y_{t-1} \mid X=x, D_{t}=0}\left(y-F_{Y_{t-1} \mid X=x, D_{t}=1}^{-1} \circ F_{Y_{t-1} \mid X=x, D_{t}=0}(v), v\right)}{f_{Y_{t-1} \mid X=x, D_{t}=1} \circ F_{Y_{t-1} \mid X=x, D_{t}=1}^{-1} \circ F_{Y_{t-1} \mid X=x, D_{t}=0}(v)},
$$

for $(y, v) \in \mathcal{Y}_{t \mid x, 1}(0) \times \mathcal{Y}_{t-1 \mid x, 0}$.

This proposition shows that the limiting process $\mathbb{Z}_{x}^{(0)}$ for the counterfactual distribution has an extra term depending on the map $\kappa_{x}$, which reflects our identification argument of the counterfactual distribution of interest as well as the contribution of estimation errors from empirical distributions. Thus the limiting distribution is not nuisance parameter free, and a bootstrap procedure can facilitate statistical inference in practice as shown in the next subsection.

Next we present the limiting process of the CQTT estimators over a range of quantile $\mathcal{T}$. Proposition 1 together with the functional delta method delivers the following theorem.

Theorem 2. Suppose that Assumption A1-A6 hold. If $F_{Y_{t}(0) \mid X, D_{t}=1}$ admits a positive continuous density $f_{Y_{t}(0) \mid X, D_{t}=1}$ on an interval $[a, b]$ containing an $\epsilon$-enlargement of the set $\left\{F_{Y_{t}(0) \mid X, D_{t}=1}^{-1}(\tau): \tau \in \mathcal{T}\right\} \subset \mathcal{Y}_{t \mid X, 1}(0)$ with $\mathcal{T} \subset(0,1)$, then, for each $x \in \mathcal{X}$,

$$
\sqrt{n}\left(\hat{\Delta}_{x}^{Q T T}(\tau)-\Delta_{x}^{Q T T}(\tau)\right) \rightsquigarrow \overline{\mathbb{Z}}_{x}^{(1)}(\tau)-\overline{\mathbb{Z}}_{x}^{(0)}(\tau),
$$


where $\left(\overline{\mathbb{Z}}_{x}^{(0)}(\tau), \overline{\mathbb{Z}}_{x}^{(1)}(\tau)\right)$ is a stochastic process in the metric space $\left(\ell^{\infty}(\mathcal{T})\right)^{2}$, given by

$$
\overline{\mathbb{Z}}_{x}^{(j)}(\tau):=\frac{\mathbb{Z}_{x}^{(j)}\left(F_{Y_{t}(j) \mid X=x, D_{t}=1}^{-1}(\tau)\right)}{f_{Y_{t}(j) \mid X=x, D_{t}=1}\left(F_{Y_{t}(j) \mid X=x, D_{t}=1}^{-1}(\tau)\right)},
$$

for $j=0,1$.

Using the result in Proposition 1 with a similar argument used in Theorem 2, one could also consider other plug-in estimators of Hadamard differentiable functionals, such as Lorenz curve and Gini coefficient and obtain their limit processes. Also, we can consider testing that $\Delta_{x}^{Q T T}(\tau)=0$ for all $\tau \in \mathcal{T}$ using the Kolmogorov-Smirnov (KS) test statistic given by $K S_{x}:=\sqrt{n} \sup _{\tau \in \mathcal{T}}\left|\hat{\Delta}_{x}^{Q T T}(\tau)\right|$. The next corollary states this result; it follows directly from Theorem 2 and the continuous mapping theorem.

Corollary 2. Suppose that the conditions of Theorem 2 hold. Under the null hypothesis $H_{0}: \Delta_{x}^{Q T T}(\tau)=0$ for all $\tau \in \mathcal{T}$, we have, for each $x \in \mathcal{X}$,

$$
K S_{x} \stackrel{d}{\rightarrow} \sup _{\tau \in \mathcal{T}}\left|\overline{\mathbb{Z}}_{x}^{(1)}(\tau)-\overline{\mathbb{Z}}_{x}^{(0)}(\tau)\right|
$$

In addition to testing for zero CQTT, one can also use this result to form asymptotic simulataneous $(1-\alpha) \%$ confidence bands for the entire CQTT process. The confidence bands are given by $\left(\hat{\Delta}_{x}^{Q T T}-c_{1-\alpha} n^{-1 / 2}, \hat{\Delta}_{x}^{Q T T}+c_{1-\alpha} n^{-1 / 2}\right)$ where $c_{1-\alpha}$ are critical values from the $\mathrm{KS}$ test. In practice, the critical values can be obtained using the bootstrap.

\subsection{Bootstrap}

The limiting processes presented in the preceding section depend on unknown nuisance parameters, some of which require nonparametric estimation and may complicate inference in finite samples. To deal with the issue of nonpivotal limit processes, we consider a resampling method called the exchangeable bootstrap (see Præstgaard and Wellner (1993) and van der Vaart and Wellner (1996)). This resampling scheme consistently estimates limit laws of relevant empirical distributions and thus with the functional delta method consistently estimates the limit process of the CQTT estimator.

For the resampling scheme, we introduce a vector of random weights $\left(W_{1}^{(d)}, \ldots, W_{n}^{(d)}\right)$ for $d=0,1$. To establish the validity of the bootstrap, we assume that the random weights satisfy the following conditions.

Assumption B. For each $d \in\{0,1\}$, let $\left(W_{1}^{(d)}, \ldots, W_{n}^{(d)}\right)$ be an $n$-dimensional vector of exchangeable, nonnegative random variables. The vectors $\left(W_{1}^{(0)}, \ldots, W_{n}^{(0)}\right)$ and $\left(W_{1}^{(1)}, \ldots, W_{n}^{(1)}\right)$ are independent of the original sample as well as each other. The vectors of random weights, depending on the size of each group, satisfy the following conditions:

$$
\max _{1 \leq i \leq n} E\left|W_{i}^{(d)}\right|^{2+\epsilon}<\infty, \quad \bar{W}_{n, x}^{(d)}:=\frac{1}{n_{x}^{(d)}} \sum_{i=1}^{n} W_{i}^{(d)} \delta_{i, x}^{(d)} \rightarrow^{p} 1, \quad \frac{1}{n_{x}^{(d)}} \sum_{i=1}^{n}\left(W_{i}^{(d)}-\bar{W}_{n, x}^{(d)}\right)^{2} \delta_{i, x}^{(d)} \rightarrow^{p} 1,
$$


for each $d \in\{0,1\}$.

As van der Vaart and Wellner (1996) explain, this resampling scheme encompasses a variety of bootstrap methods, such as the empirical bootstrap, subsampling, wild bootstrap and so on. This condition is employed in Chernozhukov et al. (2013a) for inference of counterfactual distributions. For the empirical application in this paper, we consider the empirical bootstrap, which corresponds to the case where the vector of random weights $\left(W_{1}^{(d)}, \ldots, W_{n}^{(d)}\right)$ follows the multinomial distribution with probabilities $\delta_{i, x}^{(d)} \cdot\left(1 / n_{x}^{(d)}, \ldots, 1 / n_{x}^{(d)}\right)$. Given each realization of random weights, we apply the estimation procedure explained in the previous section and estimate the parameters of interest. As we show below, the repetition of the bootstrap leads to asymptotically valid inference. The other types of resampling methods, such as weighted bootstrap or subsampling, also can be considered under the same framework and shown to be valid. In our empirical application, the sample size is moderate and our estimation procedure uses empirical distribution functions and thus the empirical bootstrap is straightforward and convenient. In the other applications, however, one might prefer weighted bootstrap if the estimation procedure is time-consuming or subsampling if the sample size is extremely large.

Given the random weights, we define the weighted bootstrap empirical distribution as

$$
\hat{F}_{Y_{s} \mid X=x, D_{t}=d}^{*}(y):=\frac{1}{n_{x}^{(d)}} \sum_{i=1}^{n} W_{i}^{(d)} 1\left\{Y_{i s} \leq y\right\} \delta_{i, x}^{(d)}
$$

for $(s, d) \in\{t-1, t\} \times\{0,1\}$. As in the previous subsection, the bootstrap distribution of the treated potential outcome $\hat{F}_{Y_{t}(1) \mid X=x, D_{t}=1}^{*}$ is given by $\hat{F}_{Y_{t} \mid X=x, D_{t}=1}^{*}$, while the bootstrap version of the counterfactual distribution is given by

$\hat{F}_{Y_{t}(0) \mid X=x, D_{t}=1}^{*}(y):=\frac{1}{n_{x}^{(0)}} \sum_{i=1}^{n} W_{i}^{(0)} 1\left\{\Delta Y_{i t}+\hat{F}_{Y_{t-1}^{*-1} \mid X=x, D_{t}=1} \circ \hat{F}_{Y_{t-1} \mid X=x, D_{t}=0}^{*}\left(Y_{i, t-1}\right) \leq y\right\} \delta_{i, x}^{(0)}$,

for $y \in \mathbb{R}$, where $\hat{F}_{Y_{t-1} \mid X=x, D_{t}=1}^{*-1}$ is the bootstrap version of the quantile function obtained through the bootstrap empirical distribution $\hat{F}_{Y_{t-1} \mid X=x, D_{t}=1}^{*}$. The bootstrap version of the CQTT process given $X_{i}=x$ is given by

$$
\hat{\Delta}_{x}^{Q T T *}(\tau):=\hat{F}_{Y_{t}(1) \mid X=x, D_{t}=1}^{*-1}(\tau)-\hat{F}_{Y_{t}(0) \mid X=x, D_{t}=1}^{*-1}(\tau),
$$

for $\tau \in \mathcal{T}$, where $\hat{F}_{Y_{t}(j) \mid X=x, D_{t}=1}^{*-1}(\tau)$ is the $\tau$ th conditional quantile of the bootstrap empirical distribution $\hat{F}_{Y_{t}(j) \mid X=x, D_{t}=1}^{*}$ of potential outcomes for $j=0,1$.

For the validity of the resampling method explained above, we need to introduce the notion of conditional weak convergence in probability, following van der Vaart and Wellner (1996). For some normed space $\mathbb{D}$, let $B L_{1}(\mathbb{D})$ denote the space of all Lipschitz continuous functions from $\mathbb{D}$ to $[-1,1]$. Given the original sample $\mathbf{D}_{n}$ with $n$ being the sample size, consider a random element $B_{n}^{*}:=g\left(\mathbf{D}_{n}, \mathbf{W}_{n}\right)$ as a function of the original sample and the random weight vector $\mathbf{W}_{n}$ generating the bootstrap draw. The bootstrap law of $B_{n}^{*}$ is said to consistently estimate the law of some tight random element $B$, or $B_{n}^{*} \rightsquigarrow^{p} B$ if

$$
\sup _{h \in B L_{1}(\mathbb{D})}\left|E_{\mathbf{W}_{n}}\left[h\left(B_{n}^{*}\right)\right]-E[h(B)]\right| \rightarrow^{p} 0,
$$


where $E_{\mathbf{W}_{n}}$ is the conditional expectation with respect to $\mathbf{W}_{n}$ given the original sample $\mathbf{D}_{n}$.

To state a preliminary result, we define empirical processes indexed by $\mathcal{Y}_{s \mid x, d}$ as

$$
\hat{G}_{s, x}^{(d) *}:=\sqrt{n}\left(\hat{F}_{Y_{s} \mid X=x, D_{t}=d}^{*}-\hat{F}_{Y_{s} \mid X=x, D_{t}=d}\right),
$$

for $(s, d) \in\{t-1, t\} \times\{0,1\}$. Also, define an empirical process indexed by $\mathcal{Y}_{t \mid x, 1}(0)$ as

$$
\tilde{G}_{t, x}^{(0) *}:=\sqrt{n}\left(\tilde{F}_{Y_{t}(0) \mid X=x, D_{t}=1}^{*}-\tilde{F}_{Y_{t}(0) \mid X=x, D_{t}=1}\right),
$$

where the empirical distribution is given by

$$
\tilde{F}_{Y_{t}(0) \mid X=x, D_{t}=1}^{*}(y):=\frac{1}{n_{x}^{(0)}} \sum_{i=1}^{n} W_{i}^{(0)} 1\left\{\Delta Y_{i t}+F_{Y_{t-1} \mid X=x, D_{t}=1}^{-1} \circ F_{Y_{t-1} \mid X=x, D_{t}=0}\left(Y_{i, t-1}\right) \leq y\right\} \delta_{i, x}^{0} .
$$

The following lemma shows that a set of the empirical processes defined above consistently estimates the tight random element defined in Lemma 1 .

Lemma 2. Suppose that Assumption $A 1-A 6$ and $B$ hold. Then, for each $x \in \mathcal{X}$,

$$
\left(\tilde{G}_{t, x}^{(0) *}, \hat{G}_{t-1, x}^{(0) *}, \hat{G}_{t, x}^{(1) *}, \hat{G}_{t-1, x}^{(1) *}\right) \rightsquigarrow{ }^{p}\left(\mathbb{V}_{x}^{(0)}, \mathbb{W}_{x}^{(0)}, \mathbb{V}_{x}^{(1)}, \mathbb{W}_{x}^{(1)}\right)
$$

in $\mathbb{S}_{x}$, where the limit processes defined in Lemma 1 .

Using this lemma, we first show that the exchangeable bootstrap provides a way to consistently estimate limit process of a pair of empirical distributions of potential outcomes. Subsequently we argue that the limit process of the CQTT estimator can be estimated, using the functional delta method for a Hadmard differentiable map. The result is summarized in the following theorem.

Theorem 3. Define $\hat{Z}_{x}^{(j) *}(y):=\sqrt{n}\left(\hat{F}_{Y_{t}(j) \mid X=x, D_{t}=1}^{*}(y)-\hat{F}_{Y_{t}(j) \mid X=x, D_{t}=1}(y)\right)$ for $j \in\{0,1\}$, $x \in \mathcal{X}$ and $y \in \mathcal{Y}_{t \mid x, 1}(j)$. Suppose that Assumption A1-A6 and B hold. Then, for each $x \in \mathcal{X}$,

$$
\left(\hat{Z}_{x}^{(0) *}, \hat{Z}_{x}^{(1) *}\right) \rightsquigarrow^{p}\left(\mathbb{Z}_{x}^{(0)}, \mathbb{Z}_{x}^{(1)}\right),
$$

and thus the exchangeable bootstrap procedure consistently estimates the law of the limit stochastic process of the CQTT:

$$
\sqrt{n}\left(\hat{\Delta}_{x}^{Q T T *}(\tau)-\hat{\Delta}_{x}^{Q T T}(\tau)\right) \rightsquigarrow^{p} \overline{\mathbb{Z}}_{x}^{(1)}(\tau)-\overline{\mathbb{Z}}_{x}^{(0)}(\tau), \quad \tau \in \mathcal{T} .
$$




\section{Monte-Carlo Simulation}

We consider a small scale Monte Carlo simulation to assess the performance of our estimator in finite samples and consider the effect of small deviations from the Copula Invariance assumption. The data generating process (DGP) for potential outcomes is given by

$$
Y_{i t}(d)=\mu(d)+\theta_{t}+v_{i}+\epsilon_{i t}
$$

In this setup, the treatment effect is constant across all quantiles and given by $\mu(1)-\mu(0)$. $\theta_{t}$ is a time fixed effect that is common across individuals; $v_{i}$ is time invariant unobserved heterogeneity that can be distributed differently across treated and untreated groups; and $\epsilon_{i t}$ are time varying unobservables. Throughout, we impose that $\theta_{t}=1$ and set $\mu(1)-\mu(0)$ to be either 1 or 0 and label this effect TE.

DGP 1: The first DGP imposes that $v_{i} \mid D=d \sim N(d, 1)$ and that $\epsilon_{i t}$ is a noise term that follows a standard normal distribution. One can show that both our model and the Change in Changes model (Athey and Imbens, 2006) hold under this setup. We use this DGP to assess the finite sample performance of our estimator using the Change in Changes model as a benchmark. We perform 1000 Monte Carlo simulations and at each iteration, we use 1000 bootstrap iterations to calculate empirical rejection frequencies given the nominal size of $5 \%$. We calculate standard errors using the empirical block bootstrap for the Change in Changes using the same sample and 1000 bootstrap iterations.

The results are presented in Table 1 . The first panel of Table 1 considers the case where the treatment effect is 0 at all quantiles. Relative to the Change in Changes models, our estimator is less biased in finite samples especially at the 0.9 th quantile. With only 100 observations, our inference procedure is somewhat undersized, but it exhibits good size properties with 200 or 500 observations. Finally, the second panel considers the case where the treatment effect is 1 at all quantiles. The power of our inference procedure increases rapidly as the sample size increases from 100 to 200 and then to 500. It also has more power at the median than at the 0.1 th or 0.9 th quantiles.

DGP 2: For the second DGP, we want to assess the effect of small deviations from the Copula Invariance assumption while the Distributional DID assumption continues to hold. To do this, we assume that

$$
\left(v_{i}, \epsilon_{i 2}, \epsilon_{i 1}\right) \mid D=d \sim N\left(0, V_{d}\right)
$$

where

$$
V_{d}=\left(\begin{array}{ccc}
1 & \rho_{d v 2} & \rho_{d v 1} \\
\rho_{d v 2} & 1 & \rho_{d 12} \\
\rho_{d v 1} & \rho_{d 12} & 1
\end{array}\right)
$$

Under this setup, $\left(Y_{i 1}(0), \Delta Y_{i 2}(0) \mid D=d\right)$ has a bivariate normal distribution with correlation parameter $\rho_{d 2}-\rho_{d 1}+\rho_{d 12}-1$. For bivariate normal distributions, the copula is Gaussian with the dependence parameter given by the correlation coefficient. For DGP 2, 
we set $\rho_{d 1}=0$ and $\rho_{d 12}=1 / 2$ both for $d=0,1$; then, we set $\rho_{d 2}=d \bar{\rho}$ and vary $\bar{\rho}$. For $\bar{\rho}=0$, the Copula Invariance assumption holds, but it is violated when $\bar{\rho} \neq 0$. For each simulation, we consider the case with $N=200$.

The results are presented in Table 2. Small violations of the Copula Invariance assumption $(\bar{\rho}=0.05$ or $\bar{\rho}=0.10)$ lead to small increases in the bias of our estimator. For example, for the 0.1 th quantile, the bias increases from 0.020 to 0.073 and 0.121 as $\bar{\rho}$ increases from 0.00 to 0.05 to 0.10 . A large increase in the violation of the Copula Invariance assumption, $\bar{\rho}=0.50$, leads to a much larger increase in the bias of our estimator for the 0.1th quantile (bias increases to 0.425 ) in our simulations. On the other hand, the results for the 0.5th quantile are almost completely insensitive to deviations from the Copula Invariance assumption. In the large deviation case, $\bar{\rho}=0.5$, the bias is very small: 0.013 ; nor does the root mean squared error change much even with large violations of the Copula Invariance assumption for the 0.5 th quantile.

\section{Empirical Application}

To illustrate our method, we consider the effect of increases in the minimum wage on the distribution of earnings. In 1996, President Bill Clinton signed a bill to increase the federal minimum wage from $\$ 4.25$ to $\$ 5.15$ per hour by September 1997 . The minimum wage did not increase again until the Fair Minimum Wage Act of 2007. The Fair Minimum Wage Act was proposed on January 5, 2007; signed on May 25, 2007 by President George W. Bush; increased the minimum wage to $\$ 5.85$ on July 24,2007 ; and in two more gradual increases settled on $\$ 7.25$ per hour in July 2009 .

We exploit the long period from 1999-2007 with a flat, national minimum wage to use state-level variation in the minimum wage to identify and estimate the effect of increasing the minimum wage on the earnings distribution. In the first quarter of 2006, for 33 states the federal minimum wage was binding. The other states had state minimum wages that were higher than the federal minimum wage. For our analysis, we take a subset of states that raised their minimum wage in the first quarter of 2007 and have a close geographic proximity to a state whose effective minimum wage is given by the federal minimum wage for the entire period. This results in a sample of 5 states that increase their minimum wage (Arizona, Colorado, Minnesota, Missouri, and North Carolina) - this is the treated group - and 14 control states (Georgia, Idaho, Iowa, Kansas, Kentucky, Nebraska, New Mexico, North Dakota, South Carolina, South Dakota, Tennessee, Utah, Virginia, and Wyoming). The large literature on estimating the effect of changes in minimum wage policies has emphasized that unconditional DID methods are not likely to be valid (Dube et al., 2010). There has also been an interest in understanding the effect of minimum wages on the distribution of earnings (Dube, 2013).

The data for the application comes from the Current Population Survey (CPS) (Flood et al. 2015). The CPS surveys roughly 140,000 individuals per month. Individuals are interviewed for four consecutive months, out of the sample for eight months, and then interviewed for four more months. Importantly for our purposes, individual earnings questions are asked in the 4th month and in the 8th month in the sample - due to the survey design, these are exactly one year apart. There are some difficulties with linking the CPS over time, but 
longitudinal identifiers are available in the IPUMS database (Drew et al., 2014). We limit the sample to individuals who have earnings greater than $\$ 10$ per week and to those that we can successfully link over time. This procedure results in a sample size of 8256 individuals (2 observations per individual) that are observed in the first two quarters of 2007 and at some point in 2006.

Next, we divide the data into 8 categories based on gender, race (white or non-white), and education attainment (college graduate or not). Summary statistics are provided in Table 3. There are several important differences between treated states and untreated states. First, in 2006, earnings in states that raised their minimum wage were 6.5 log points (statistically significant) higher, on average, than in states that did not raise their minimum wage. This provides some evidence that cross sectional comparisons of earnings distributions, at least without adjusting for covariates, are likely to lead to upwardly biased estimates of the effect of the minimum wage on the earnings distribution. Second, there are differences in the covariates across treated and untreated states. While the fraction of male individuals is similar across treated and untreated states, individuals in states that raised their minimum wage are more likely to be white and more likely to have a college degree. If the path of earnings, in the absence of changes in minimum wage policy, depends on race and education, then it will be important to control for these covariates in the analysis; similarly, if the dependence between the change in outcomes over time and the initial level of outcomes (both in the absence of changes in minimum wage policy) depends on these variables, then it will be important to condition on these variables. Our method makes it possible to account for both of these complications.

To evaluate the effect of minimum wage increases across each subgroup, first we test whether the CQTT is 0 across all quantiles using the Kolmogorov-Smirnov test statistic. In order to do this, we estimate the CQTT for each group over a fine grid of $\tau$ from 0.05 to 0.95 by 0.01 and using 1000 boostrap iterations to calculate the critical values of the test. These results are available in Table 4. For only 3 out of 8 of the race-gender-education subgroups are we able to reject the null that the distribution of earnings is the same due to the change in the minimum wage policy. We reject the null of no effect at any quantile for (i) white, female, college graduates; (ii) non-white, male, non-college, and (iii) non-white, female, non-college. Interestingly, the groups for which we can reject the null tend to have lower earnings than other groups - this seems intuitive as the minimum wage is binding only at the lower part of the earnings distribution. Table 4 also provides estimates of the CQTT for each subgroup at the $0.1,0.5$, and 0.9 quantiles with pointwise standard errors computed with 1000 bootstrap iterations reported.

Second, Figure 1 plots the CQTT for each subgroup as well as $95 \%$ confidence bands. The confidence bands are obtained by inverting the Kolmogrov-Smirnov test mentioned above. For each of the groups that we reject the null of no effect, we only find statistically significant results at the lower part of the distribution which also corresponds to our intuition about the effects of increasing the minimum wage. Interestingly, the effect of the minimum wage on earnings appears to be negative. This result may appear surprising, but it should be remembered that earnings mixes both wages and hours. Thus, even if the minimum wage lifts wages in the lower part of the distribution, our result could be be explained by a decrease 
in hours due to minimum wage increases ${ }^{1}$

\section{Conclusion}

This paper has considered identifying and estimating the Conditional Quantile Treatment Effect on the Treated under a Distributional DID assumption when only two periods of data are available. We have developed uniform confidence intervals for the CQTT and shown the validity of a bootstrap procedure for computing confidence bands. Finally, we estimated conditional quantile treatment effects for states that increased their minimum wage.

Methodologically, the key innovation is to recover the unknown dependence between the change and initial level of untreated potential outcomes for the treated group from the observed dependence from the untreated group. Combining this condition with a distributional extension of the most common mean DID assumption results in point identification of the counterfactual conditional distribution of untreated potential potential outcomes for the treated group; and, therefore, to identification of the CQTT. There are many examples in finance, auction models, and duration models where identification depends on an unknown copula. The idea of replacing an unknown copula with one observed for another group may prove to be a fruitful line of research in those cases.

\footnotetext{
${ }^{1}$ As a robustness check, we also compare the change in the 10th percentile of earnings between 2007 and 2006 for the treated group to the same change for the untreated group. For the treated group, the 10th percentile of log earnings increased by $3.0 \mathrm{log}$ points, but the 10th percentile for the untreated group increased by $10.5 \log$ points. This result is in line with our results that say that the distribution of earnings for subgroups that experienced an effect of the minimum wage tended to be worse than the distribution of earnings would have been absent changes in the minimum wage policy.
} 


\section{References}

AbAdie, A. (2003): "Semiparametric Instrumental Variable Estimation of Treatment Response Models," Journal of econometrics, 113, 231-263.

(2005): "Semiparametric Difference-in-Differences Estimators," The Review of Economic Studies, 72, 1-19.

Abadie, A., J. Angrist, And G. Imbens (2002): "Instrumental Variables Estimates of the Effect of Subsidized Training on the Quantiles of Trainee Earnings," Econometrica, $70,91-117$.

Abrevaya, J. And C. M. Dahl (2008): "The Effects of Birth Inputs on Birthweight," Journal of Business \&5 Economic Statistics, 26, 379-397.

Amemiya, T. (1973): "Regression Analysis when the Dependent Variable is Truncated Normal," Econometrica, 41, 997-1016.

(1985): Advanced econometrics, Harvard university press.

Arellano, M. And S. Bonhomme (2016): "Nonlinear Panel Data Estimation via Quantile Regressions," The Econometrics Journal, 19.

Athey, S. And G. Imbens (2006): "Identification and Inference in Nonlinear Differencein-Differences Models," Econometrica, 74, 431-497.

Bertrand, M., E. Duflo, and S. Mullainathan (2004): "How Much Should We Trust Differences-in-Differences Estimates?" The Quarterly Journal of Economics, 119, 249-275.

Bitler, M. P., J. B. Gelbach, And H. W. Hoynes (2006): "Welfare Reform and Children's Living Arrangements," Journal of Human Resources, 41, 1-27.

(2008): "Distributional Impacts of the Self-Sufficiency Project," Journal of Public Economics, 92, 748-765.

Bitler, M. P., H. W. Hoynes, And T. Domina (2014): "Experimental Evidence on Distributional Effects of Head Start," Worker Paper, National Bureau of Economic Research.

Callaway, B. And T. Li (2015): "Quantile Treatment Effects in Difference in Differences Models with Panel Data," Unpublished manuscript, Vanderbilt University.

Canay, I. (2011): "A Simple Approach to Quantile Regression for Panel Data," The Econometrics Journal, 14, 368-386.

Chernozhukov, V., I. Fernandez-Val, S. Hoderlein, H. Holzmann, And W. NEWEy (2015): "Nonparametric Identification in Panels using Quantiles," Journal of Econometrics, 188, 378-392.

Chernozhukov, V., I. Fernández-Val, and B. Melly (2013a): "Inference on Counterfactual Distributions," Econometrica, 81, 2205-2268.

Chernozhukov, V., I. FernandezVal, J. Hahn, and W. Newey (2013b): "Average and Quantile Effects in Nonseparable Panel Models," Econometrica, 81, 535-580. 
Chernozhukov, V. And C. Hansen (2005): "An IV Model of Quantile Treatment Effects," Econometrica, 73, 245-261.

(2006): "Instrumental Quantile Regression Inference for Structural and Treatment Effect Models," Journal of Econometrics, 132, 491-525.

_ (2013): "Quantile Models with Endogeneity," Annual Review of Economics, 5, 57-81.

Chetverikov, D., B. Larsen, and C. Palmer (2016): "IV Quantile Regression for Group-Level Treatments, With an Application to the Distributional Effects of Trade," Econometrica, 84, 809-833.

D'Haultfoeuille, X., S. Hoderlein, and Y. Sasaki (2015): "Nonlinear Differencein-Differences in Repeated Cross Sections with Continuous Treatments," Working Paper.

DJebbari, H. And J. Smith (2008): "Heterogeneous Impacts in PROGRESA," Journal of Econometrics, 145, 64-80.

Drew, J. A. R., S. Flood, And J. R. Warren (2014): "Making Full Use of the Longitudinal Design of the Current Population Survey: Methods for Linking Records across 16 Months," Journal of Economic and Social Measurement, 39, 121.

Dube, A. (2013): "Minimum Wages and the Distribution of Family Incomes," Unpublished manuscript.

Dube, A., T. W. Lester, And M. Reich (2010): "Minimum Wage Effects across State Borders: Estimates using Contiguous Counties," The Review of Economics and Statistics, 92, 945-964.

Eren, O. And S. OzBekLik (2014): "Who Benefits from Job Corps? A Distributional Analysis of an Active Labor Market Program," Journal of Applied Econometrics, 29, 586611.

FAN, Y. AND Z. YU (2012): "Partial Identification of Distributional and Quantile Treatment Effects in Difference-in-Differences Models," Economics Letters, 115, 511-515.

Farber, H. S. (1997): "The Changing Face of Job Loss in the United States, 1981-1995," Brookings Papers on Economic Activity. Microeconomics, 55-142.

FirPo, S. (2007): "Efficient Semiparametric Estimation of Quantile Treatment Effects," Econometrica, 75, 259-276.

Firpo, S., N. M. Fortin, And T. Lemieux (2009): "Unconditional Quantile Regressions," Econometrica, 953-973.

Flood, S., M. King, S. Ruggles, and J. R. Warren (2015): "Integrated Public Use Microdata Series, Current Population Survey: Version 4.0 [dataset]," Minneapolis: University of Minnesota.

Frölich, M. And B. Melly (2013): "Unconditional Quantile Treatment Effects under Endogeneity," Journal of Business 83 Economic Statistics, 31, 346-357.

Galvao, A. F. And K. Kato (2014): "Estimation and Inference for Linear Panel Data Models Under Misspecification When Both $\mathrm{n}$ and T are Large," Journal of Business 83 Economic Statistics, 32, 285-309. 
Galvao, A. F., C. Lamarche, and L. R. Lima (2013): "Estimation of Censored Quantile Regression for Panel Data With Fixed Effects," Journal of the American Statistical Association, 108, 1075-1089.

Graham, B. S., J. Hahn, A. Poirier, and J. L. Powell (2015): "Quantile Regression with Panel Data," Tech. rep., National Bureau of Economic Research.

Gronau, R. (1974): "Wage Comparisons-A Selectivity Bias," Journal of Political Economy, $82,1119-1143$.

Heckman, J., H. Ichimura, J. Smith, And P. Todd (1998): "Characterizing Selection Bias Using Experimental Data," Econometrica, 66, 1017-1098.

Heckman, J. J. (1974): "Shadow Prices, Market Wages, and Labor Supply," Econometrica, 42, 679-94.

Kato, K., A. Galvao, And G. V. Montes-Rojas (2012): "Asymptotics for Panel Quantile Regression Models with Individual Effects," Journal of Econometrics.

Khan, S., M. Ponomareva, and E. Tamer (2016): "Identification of Panel Data Models with Endogenous Censoring," Journal of Econometrics, 194, 57-75.

Koenker, R. (2005): Quantile Regression, 38, Cambridge university press.

Koenker, R. And G. Bassett (1978): "Regression Quantiles," Econometrica: Journal of the Econometric Society, 33-50.

Kosorok, M. R. (2007): Introduction to Empirical Processes and Semiparametric Inference, Springer Science \& Business Media.

Li, T. AND T. OKA (2015): "Set Identification of the Censored Quantile Regression Model for Short Panels with Fixed Effects," Journal of Econometrics, 188, 363-377.

Madrian, B. C. And L. J. Lefgren (2000): "An Approach to Longitudinally Matching Current Population Survey (CPS) Respondents," Journal of Economic and Social Measurement, 26, 31-62.

Melly, B. And G. Santangelo (2015): "The Changes-in-Changes Model with Covariates," Working Paper.

Prestgaard, J. And J. A. Wellner (1993): "Exchangeably Weighted Bootstraps of the General Empirical Process," The Annals of Probability, 2053-2086.

Riddell, W. C. AND X. Song (2011): "The Impact of Education on Unemployment Incidence and Re-employment Success: Evidence from the US Labour Market," Labour Economics, 18, 453-463.

Rosen, A. (2012): "Set Identification via Quantile Restrictions in Short Panels," Journal of Econometrics, 166, 127-137.

Rosenblatt, M. (1952): "Remarks on a Multivariate Transformation," The Annals of Mathematical Statistics, 470-472.

Rothe, C. (2012): "Partial Distributional Policy Effects," Econometrica, 80, 2269-2301.

VAN DER VAART, A. W. AND J. A. Wellner (1996): Weak Convergence and Empirical Processes with Applications to Statistics, Springer. 
(2007): "Empirical Processes Indexed by Estimated Functions." in Asymptotics: particles, processes and inverse problems. Festschrift for Piet Groeneboom., Institute of Mathematical Statistics, 234-252. 


\section{Appendix}

In Appendix, we use $\|\cdot\|$ to denote the Euclidean norm for vectors.

Proof of Theorem 1. Let $x \in \mathcal{X}$ be fixed. For every $y \in \operatorname{supp}\left(Y_{i t}(0) \mid X_{i}=x, D_{i t}=1\right)$, we can write that $F_{Y_{t}(0) \mid X=x, D_{t}=1}(y)=\operatorname{Pr}\left\{\Delta Y_{i t}(0)+Y_{i, t-1}(0) \leq y \mid X_{i}=x, D_{i t}=1\right\}$. Define

$$
U_{i}^{d}:=F_{\Delta Y_{t}(0) \mid X=x, D_{t}=d}\left(\Delta Y_{i t}(0)\right) \text { and } V_{i}^{d}:=F_{Y_{t-1}(0) \mid X=x, D_{t}=d}\left(Y_{i, t-1}(0)\right) \text {, }
$$

for $d \in\{0,1\}$. Under Assumption A4, we have

$$
\Delta Y_{i t}(0)=F_{\Delta Y_{t}(0) \mid X=x, D_{t}=d}^{-1}\left(U_{i}^{d}\right) \text { and } Y_{i, t-1}(0)=F_{Y_{t-1}(0) \mid X=x, D_{t}=d}^{-1}\left(V_{i}^{d}\right),
$$

almost surely (see Rosenblatt, 1952). It follows that

$F_{Y_{t}(0) \mid X=x, D_{t}=1}(y)=\operatorname{Pr}\left\{F_{\Delta Y_{t}(0) \mid X=x, D_{t}=1}^{-1}\left(U_{i}^{1}\right)+F_{Y_{t-1}(0) \mid X=x, D_{t}=1}^{-1}\left(V_{i}^{1}\right) \leq y \mid X_{i}=x, D_{i t}=1\right\}$.

For each $d \in\{0,1\}$, the joint distribution of $\left(U_{i}^{d}, V_{i}^{d}\right)$ conditional on $\left(X_{i}, D_{i t}\right)=(x, d)$ is given by a conditional copula $C_{\Delta Y_{t}(0), Y_{t-1}(0) \mid X=x, D_{t}=d}$, which is invariant with respect to the conditional variable $D_{i t}$ under Assumption A3. Thus we have

$F_{Y_{t}(0) \mid X=x, D_{t}=1}(y)=\operatorname{Pr}\left\{F_{\Delta Y_{t}(0) \mid X=x, D_{t}=1}^{-1}\left(U_{i}^{0}\right)+F_{Y_{t-1}(0) \mid X=x, D_{t}=1}^{-1}\left(V_{i}^{0}\right) \leq y \mid X_{i}=x, D_{i t}=0\right\}$.

Under Assumption A2, $F_{\Delta Y_{t}(0) \mid X=x, D_{t}=1}^{-1}(\cdot)=F_{\Delta Y_{t}(0) \mid X=x, D_{t}=0}^{-1}(\cdot)$, which with A2 yields that $F_{\Delta Y_{t}(0) \mid X=x, D_{t}=1}^{-1}\left(U_{i}^{0}\right)=\Delta Y_{i t}(0)$, almost surely. Also, using the relation in (A1) we can show that $F_{Y_{t-1}(0) \mid X=x, D_{t}=1}^{-1}\left(V_{i}^{0}\right)=F_{Y_{t-1}(0) \mid X=x, D_{t}=1}^{-1} \circ F_{Y_{t-1}(0) \mid X=x, D_{t}=0}\left(Y_{i, t-1}(0)\right)$, almost surely. Hence, the desired result follows.

Proof of Corollary 1 . Let $x \in \mathcal{X}$ be fixed. Given that the data generating process satisfies Assumption A1-A4, the result in Theorem 1 holds and we have

$F_{Y_{t}(0) \mid X=x, D_{t}=1}(y)=\operatorname{Pr}\left\{\Delta Y_{i t}(0)+F_{Y_{t-1} \mid X=x, D_{t}=1}^{-1} \circ F_{Y_{t-1} \mid X=x, D_{t}=0}\left(Y_{i, t-1}\right) \leq y \mid X_{i}=x, D_{i t}=0\right\}$, for $y \in \operatorname{supp}\left(Y_{i t}(0) \mid X_{i}=x, D_{i t}=1\right)$. Because of the repeated cross section, we cannot identify the term $\Delta Y_{i t}(0):=Y_{i t}(0)-Y_{i, t-1}(0)$ from the observed outcomes of the untreated group. Under the rank invariance assumption, however, we have

$$
F_{Y_{t}(0) \mid X=x, D_{t}=0}\left(Y_{i t}(0)\right)=F_{Y_{t-1}(0) \mid X=x, D_{t}=0}\left(Y_{i, t-1}(0)\right),
$$

where the distributions $F_{Y_{t}(0) \mid X=x, D_{t}=0}$ and $F_{Y_{t-1}(0) \mid X=x, D_{t}=0}$ of potential outcomes, can be identified by the distributions $F_{Y_{t} \mid X=x, D_{t}=0}$ and $F_{Y_{t-1} \mid X=x, D_{t}=0}$ of observed outcomes, respectively. Thus, we can identify $\Delta Y_{i t}(0)$ for individuals with $X_{i}=x$ and $D_{i t}=0$ by

$$
\widetilde{\Delta Y_{i t}}(0):=F_{Y_{t} \mid X=x, D_{t}=0}^{-1} \circ F_{Y_{t-1} \mid X=x, D_{t}=0}\left(Y_{i, t-1}\right)-Y_{i, t-1} \text {. }
$$

This leads to the desired result.

To derive the limiting distribution of the estimator for the CQTT, we present two technical lemmas concerning the Hadamard differentiability. We introduce a setup and notations used in the these lemmas. Let $F_{0}:=\left(G_{0}, H_{0}\right)$ with $G_{0}$ and $H_{0}$ being distribution functions having a compact support $\mathcal{V} \subset \mathbb{R}$ and a density function $g_{0}$ and $h_{0}$, respectively. Consider a pair of continuous random variables $\left(V_{1}, V_{2}\right)$ taking values on $\mathcal{V} \times \mathcal{V}$ with the joint distribution $F_{V_{1} V_{2}}$ having a density $f_{V_{1} V_{2}}$ as well as the marginal distributions $F_{V_{j}}$ having a density $f_{V_{j}}$ for $j=0,1$. We suppose that the conditional distribution $F_{V_{1} \mid V_{2}}$ has a continuous density function $f_{V_{1} \mid V_{2}}$ uniformly bounded away from 0 and $\infty$. 
Lemma A1. Let $\mathbb{D}:=(C(\mathcal{V}))^{2}$ and define the map $\psi: \mathbb{D}_{\psi} \subset \mathbb{D} \mapsto \ell^{\infty}(\mathcal{V})$, given by

$$
\psi(F):=G^{-1} \circ H
$$

for $F:=(G, H) \in \mathbb{D}_{\psi}$, where $\mathbb{D}_{\psi}:=\mathbb{E} \times \mathbb{E}$ with $\mathbb{E}$ denoting the set of all distributions functions having a strictly positive, bounded density. Then, the map $\psi$ is Hadamard differentiable at $F_{0}$ tangentially to $\mathbb{D}$. Its derivative at $F_{0}$ in $\gamma:=\left(\gamma_{1}, \gamma_{2}\right) \in \mathbb{D}$ is given by

$$
\psi_{F_{0}}^{\prime}(\gamma)=\frac{\gamma_{2}-\gamma_{1} \circ G_{0}^{-1} \circ H_{0}}{g_{0} \circ G_{0}^{-1} \circ H_{0}} .
$$

Proof. To prove the assertion, we first represent $\psi$ as a composition map. Let $\mathbb{D}_{\psi_{2}}:=$ $\mathbb{E}^{-} \times C(\mathcal{V})$, where $\mathbb{E}^{-}$denotes the set of generalized inverse of all functions in $\mathbb{E}$. Define the maps $\psi_{1}: \mathbb{D}_{\psi} \mapsto \mathbb{D}_{\psi_{2}}$ and $\psi_{2}: \mathbb{D}_{\psi_{2}} \mapsto \ell^{\infty}(\mathcal{V})$, given by

$$
\psi_{1}(\Gamma):=\left(\Gamma_{1}^{-1}, \Gamma_{2}\right) \quad \text { and } \quad \psi_{2}(\Lambda):=\Lambda_{1} \circ \Lambda_{2},
$$

for $\Gamma:=\left(\Gamma_{1}, \Gamma_{2}\right) \in \mathbb{D}_{\psi}$ and $\Lambda:=\left(\Lambda_{1}, \Lambda_{2}\right) \in \mathbb{D}_{\psi_{2}}$. Then we can write $\psi=\psi_{2} \circ \psi_{1}$.

For the map $\psi_{1}$, Lemma 3.9.23(ii) of van der Vaart and Wellner (1996) implies that if $\Gamma$ has a derivative denoted by $\Gamma^{\prime}$, then the map $\psi_{1}$ is Hadamard differentiable at $\Gamma$ tangentially to $\mathbb{D}$. Its derivative at $\Gamma$ in $\gamma:=\left(\gamma_{1}, \gamma_{2}\right) \in \mathbb{D}$ is given by

$$
\psi_{1, \Gamma}^{\prime}(\gamma):=\left(-\left(\gamma_{1} / \Gamma_{1}^{\prime}\right) \circ \Gamma_{1}^{-1}, \gamma_{2}\right) .
$$

In terms of the map $\psi_{2}$, Lemma 3.9.27 of van der Vaart and Wellner (1996) implies that $\psi_{2}$ is Hadamard differentiable at $\Lambda$ tangentially to $C([0,1]) \times \ell^{\infty}(\mathcal{V})$. Its derivative at $\Lambda$ in $\lambda:=\left(\lambda_{1}, \lambda_{2}\right) \in C([0,1]) \times \ell^{\infty}(\mathcal{V})$ is given by

$$
\psi_{2, \Lambda}^{\prime}(\lambda):=\lambda_{1} \circ \Lambda_{2}+\Lambda_{1, \Lambda_{2}}^{\prime} \lambda_{2} .
$$

Lemma 3.9.3 of van der Vaart and Wellner (1996) with Hadamard derivatives of the maps $\psi_{1}$ and $\psi_{2}$ yields that $\psi_{F_{0}}^{\prime}(\gamma)=\psi_{2,\left(G_{0}^{-1}, H_{0}\right)}^{\prime} \circ \psi_{1, F_{0}}^{\prime}(\gamma)$ for $\gamma \in \mathbb{D}$, where

and

$$
\psi_{1, F_{0}}^{\prime}(\gamma)=\left(-\left(\gamma_{1} / g_{0}\right) \circ G_{0}^{-1}, \gamma_{2}\right),
$$

$$
\psi_{2,\left(G_{0}^{-1}, H_{0}\right)}^{\prime}(\lambda)=\lambda_{1} \circ H_{0}+\frac{\lambda_{2}}{g_{0} \circ G_{0}^{-1} \circ H_{0}},
$$

because $\partial G_{0}^{-1}(\tau) / \partial \tau=1 /\left(g_{0} \circ G_{0}^{-1}(\tau)\right)$. Hence the desired result follows.

Lemma A2. Let $\mathbb{D}:=(C(\mathcal{V}))^{2}$ and let $\mathcal{W}$ be a compact subset of $\mathbb{R}$. Define the map $\phi: \mathbb{D}_{\phi} \subset \mathbb{D} \mapsto \ell^{\infty}(\mathcal{W})$, given by

$$
\phi(F)(w):=\operatorname{Pr}\left\{V_{1}+G^{-1} \circ H\left(V_{2}\right) \leq w\right\},
$$

for $F:=(G, H) \in \mathbb{D}_{\phi}$ and for $w \in \mathcal{W}$, where $\mathbb{D}_{\phi}:=\mathbb{E} \times \mathbb{E}$ with $\mathbb{E}$ being the set of all distributions functions having a strictly positive, bounded density. Then, the map $\phi$ is Hadamard differentiable at $F_{0}$ tangentially to $\mathbb{D}$. Its derivative at $F_{0}$ in $\gamma:=\left(\gamma_{1}, \gamma_{2}\right) \in \mathbb{D}$ is given by

$$
\phi_{F_{0}}^{\prime}(\gamma)(w):=\int\left(\gamma_{2}\left(v_{2}\right)-\gamma_{1} \circ G_{0}^{-1} \circ H_{0}\left(v_{2}\right)\right) \frac{f_{V_{1} V_{2}}\left(w-G_{0}^{-1} \circ H_{0}\left(v_{2}\right), v_{2}\right)}{g_{0} \circ G_{0}^{-1} \circ H_{0}\left(v_{2}\right)} d v_{2} .
$$

Proof. To prove the assertion, we represent $\phi$ as a composition map. Define $\psi: \mathbb{D}_{\phi} \rightarrow$ $\mathbb{D}_{\pi}$ as in the proceeding lemma, where $\mathbb{D}_{\pi}$ denotes the set of all functions $F^{-1} \circ G$ for $\left(F^{-1}, G\right) \in \mathbb{E}^{-} \times \mathbb{E}$ with $\mathbb{E}^{-}$and $\mathbb{E}$ defined in the proof of the proceeding lemma. Define the 
$\operatorname{map} \pi: \mathbb{D}_{\pi} \mapsto \ell^{\infty}(\mathcal{W})$, given by

$$
\pi(\Xi)(w):=\int F_{V_{1} \mid V_{2}}\left(w-\Xi\left(v_{2}\right) \mid v_{2}\right) d F_{V_{2}}\left(v_{2}\right)
$$

for $w \in \mathcal{W}$. Since we can write $\phi(F)(w)=\int F_{V_{1} \mid V_{2}}\left(w-G^{-1} \circ H\left(v_{2}\right) \mid v_{2}\right) d F_{V_{2}}\left(v_{2}\right)$ for $F \in \mathbb{D}$ and $w \in \mathcal{W}$, we can show that $\phi=\pi \circ \psi$.

We wish to show that $\pi$ has a Hadamard derivative at $\Xi \in \mathbb{D}_{\pi}$ tangentially to $\mathbb{D}$ with derivative at $\Xi$ in $\xi \in \mathbb{D}$

$$
\pi_{\Xi}^{\prime}(\xi)(w)=\int \xi\left(v_{2}\right) f_{V_{1} \mid V_{2}}\left(w-\Xi\left(v_{2}\right) \mid v_{2}\right) d F_{V_{2}}\left(v_{2}\right) .
$$

Consider any sequence $t_{k}>0$ and $\Xi_{k} \in \mathbb{D}_{\pi}$ for $k \in \mathbb{N}$ such that $t_{k} \searrow 0$ and $\xi_{k}:=\left(\Xi_{k}-\right.$ $\Xi) / t_{k} \rightarrow \xi$ in $\mathbb{D}$ as $k \rightarrow \infty$. We have

$$
F_{V_{1} \mid V_{2}}\left(w-\Xi_{k}\left(v_{2}\right) \mid v_{2}\right)-F_{V_{1} \mid V_{2}}\left(w-\Xi\left(v_{2}\right) \mid v_{2}\right)=t_{k} \xi_{k}\left(v_{2}\right) \int_{0}^{1} f_{V_{1} \mid V_{2}}\left(w-\Xi\left(v_{2}\right)-r t_{k} \xi_{k}\left(v_{2}\right) \mid v_{2}\right) d r .
$$

It follows that

$$
\begin{aligned}
\frac{\pi\left(\Xi_{k}\right)-\pi(\Xi)}{t_{k}}-\pi_{\Xi}^{\prime}(\xi)= & \int\left(\xi_{k}\left(v_{2}\right)-\xi\left(v_{2}\right)\right) f_{V_{1} \mid V_{2}}\left(\cdot-\Xi\left(v_{2}\right) \mid v_{2}\right) d F_{V_{2}}\left(v_{2}\right) \\
& +\int \xi_{k}\left(v_{2}\right) D_{k}\left(\cdot, v_{2}\right) d F_{v_{2}}\left(v_{2}\right),
\end{aligned}
$$

where $D_{k}\left(w, v_{2}\right):=\int_{0}^{1}\left\{f_{V_{1} \mid V_{2}}\left(w-\Xi\left(v_{2}\right)-r t_{k} \xi_{k}\left(v_{2}\right) \mid v_{2}\right)-f_{V_{1} \mid V_{2}}\left(w-\Xi\left(v_{2}\right) \mid v_{2}\right)\right\} d r$. Since $f_{V_{1} \mid V_{2}}$ is uniformly continuous and $\xi_{k}$ is uniformly bounded, $\lim _{k \rightarrow \infty}\left\|D_{k}\right\|_{\mathcal{W} \times \mathcal{V}}=0$ and thus the second term on the above display converges to 0 as $k \rightarrow \infty$. Also the first term on the above display converges to zero because $f_{V_{2} \mid V_{1}}$ is uniformly bounded and $\left\|\xi_{k}-\xi\right\|_{\infty} \rightarrow 0$ as $k \rightarrow \infty$. Thus the map $\pi$ has the Hadamard derivative as stated.

Lemma 3.9.3 of van der Vaart and Wellner (1996) shows that $\phi_{F_{0}}^{\prime}(\gamma)=\pi_{G_{0}^{-1} \circ H_{0}}^{\prime} \circ \psi_{F_{0}}^{\prime}(h)$, which together with the Hadamard derivative of $\pi$ in $\mathrm{A} 3$ and the one of $\psi$ in Lemma A1. yields

$$
\phi_{F_{0}}^{\prime}(\gamma)(w)=\int \frac{\gamma_{2}\left(v_{2}\right)-\gamma_{1} \circ G_{0}^{-1} \circ H_{0}\left(v_{2}\right)}{g_{0} \circ G_{0}^{-1} \circ H_{0}\left(v_{2}\right)} f_{V_{1} \mid V_{2}}\left(w-G_{0}^{-1} \circ H_{0}\left(v_{2}\right) \mid v_{2}\right) d F_{V_{2}}\left(v_{2}\right) .
$$

Hence the desired result follows.

Define $\tilde{V}:=V_{1}+G_{0}^{-1} \circ H_{0}\left(V_{2}\right)$. We additionally assume that $\tilde{V}$ is distributed over a compact space $\mathcal{V}$ with a distribution $F_{\tilde{V}}$ and a continuous density $f_{\tilde{V}}$ uniformly bounded away from 0 and $\infty$. We consider random sample $\left\{\left(V_{1 i}, V_{2 i}\right)\right\}_{i=1}^{n}$ of $n$ independent copies of $\left(V_{1}, V_{2}\right)$ and let $\tilde{V}_{i}:=V_{1 i}+G_{0}^{-1} \circ H_{0}\left(V_{2 i}\right)$. We set $F_{n}:=\left(G_{n}, H_{n}\right)$ to denote a random element of $\left(\ell^{\infty}(\mathcal{V})\right)^{2}$ as a consistent estimator for $F_{0}$. For $F=(G, H) \in(C(\mathcal{V}))^{2}$ and $w \in \mathcal{W}$, define a functional taking values at $\ell^{\infty}(\mathcal{W})$ :

$$
\phi_{n}(F)(w):=n^{-1} \sum_{i=1}^{n} 1\left\{V_{1 i}+G^{-1} \circ H\left(V_{2 i}\right) \leq w\right\},
$$

and the empirical process indexed by $F \in\left(\ell^{\infty}(\mathcal{V})\right)^{2}$ :

$$
\nu_{n}(F):=\sqrt{n}\left(\phi_{n}(F)-\phi(F)\right) .
$$

The lemma below is proven, along the line of Theorem 2.3 of van der Vaart and Wellner (2007) with some modification. 
Lemma A3. Suppose that $\sqrt{n}\left(F_{n}-F_{0}\right)$ converges in distribution to a tight, random element with values in $\left(\ell^{\infty}(\mathcal{V})\right)^{2}$. Then,

$$
\sup _{w \in \mathcal{W}}\left|\nu_{n}\left(F_{n}\right)-\nu_{n}\left(F_{0}\right)\right|(w)=o_{p}(1) .
$$

Proof. Because the set $\mathcal{W}$ is compact, it suffices to shows that the assertions holds for each $w \in \mathcal{W}$. Also, from the definition of $\phi_{n}$ in $(\mathrm{A} 4)$, both $\phi_{n}(F)$ and $\nu_{n}(F)$ can be considered as functions of $\Xi:=G^{-1} \circ F \in \ell^{\infty}(\mathcal{V})$. Letting $\bar{\nu}_{n}(\Xi)=\nu_{n}(F)$, we show that, for each $w \in \mathcal{W}$,

$$
\left|\bar{\nu}_{n}\left(\Xi_{n}\right)-\bar{\nu}_{n}\left(\Xi_{0}\right)\right|(w)=o_{p}(1)
$$

where $\Xi_{n}:=G_{n}^{-1} \circ F_{n}$ and $\Xi_{0}:=G_{0}^{-1} \circ F_{0}$. Let $w \in \mathcal{W}$ be fixed and choose an arbitrary small $\epsilon>0$. Suppose that $\sqrt{n}\left(F_{n}-F_{0}\right)$ converges in distribution to some tight random element. Then, by the functional delta method and Hadamard differentiability, Lemma A1 implies

$$
\xi_{n}:=\sqrt{n}\left(\Xi_{n}-\Xi_{0}\right) \rightsquigarrow \xi_{\infty},
$$

in $\ell^{\infty}(\mathcal{V})$ for some tight random element $\xi_{\infty}$. Then there exists a compact set $S \subset \ell^{\infty}(\mathcal{V})$ such that $\operatorname{Pr}\left\{\xi_{\infty} \notin S\right\} \leq \epsilon / 2$, and also $\lim _{\sup } \rightarrow \infty \operatorname{Pr}\left\{\xi_{n} \notin S^{\delta / 4}\right\} \leq \epsilon / 2$ for any $\delta>0$, where $S^{\delta / 4}$ is the $\delta / 4$-enlargement set of $S$. Because $S$ is compact, for any $\delta>0$ there exists a finite set $\left\{\xi^{(1)}, \ldots, \xi^{(J)}\right\} \subset S$ with $J=J(\delta)$ such that $\sup _{\xi \in S} \min _{1 \leq j \leq J}\left\|\xi-\xi^{(j)}\right\|_{\infty}<\delta / 4$. It follows that, for any $\delta>0$,

$$
\operatorname{Pr}\left\{\min _{1 \leq j \leq J}\left\|\xi_{n}-\xi^{(j)}\right\|_{\infty} \geq \delta / 2\right\} \leq \operatorname{Pr}\left\{\xi_{n} \notin S^{\delta / 4}\right\} \leq \epsilon / 2
$$

for a sufficiently large $n$.

In the view of the compactness of $\mathcal{V}$, for every $\eta>0$, there is a finite set $\left\{v_{1}, \ldots, v_{K}\right\} \subset \mathcal{V}$ with $K=K(\eta)$ such that $\sup _{v \in \mathcal{V}} \min _{1 \leq k \leq K}\left|v-v_{k}\right|<\eta$. Define the map $\Pi_{\delta}: \mathcal{V} \mapsto\left\{v_{k}\right\}_{k=1}^{K}$ such that $\left|v-\Pi_{\eta}(v)\right| \leq \eta$ for every $v \in \mathcal{V}$. Theorem 1.5.7 of van der Vaart and Wellner (1996) with (A5) implies that for any $\delta>0$, there exists $\eta>0$ such that, for a sufficiently large $n$,

$$
\operatorname{Pr}\left\{\left\|\xi_{n}-\xi_{n} \circ \Pi_{\eta}\right\|_{\infty}>\delta / 2\right\}<\epsilon / 2 .
$$

It follows from (A6) and A7 that, for a sufficiently large $n$,

$$
\operatorname{Pr}\left\{\min _{1 \leq j \leq J}\left\|\xi_{n}-\xi^{(j)} \circ \Pi_{\eta}\right\|_{\infty}>\delta\right\}<\epsilon,
$$

which yields that, given a set $\mathcal{M}_{j, \eta}(\delta):=\left\{\xi \in \ell^{\infty}(\mathcal{V}):\left\|\xi-\xi^{(j)} \circ \Pi_{\eta}\right\|_{\infty} \leq \delta\right\}$, we have $\operatorname{Pr}\left\{\left|\bar{\nu}_{n}\left(\Xi_{n}\right)-\bar{\nu}_{n}\left(\Xi_{0}\right)\right|(w) \geq \epsilon\right\} \leq \sum_{j=1}^{J} \operatorname{Pr}\left\{\sup _{\xi \in \mathcal{M}_{j, \eta}(\delta)}\left|\bar{\nu}_{n}\left(\Xi_{0}+n^{-1 / 2} \xi\right)-\bar{\nu}_{n}\left(\Xi_{0}\right)\right|(w) \geq \epsilon\right\}+\epsilon$.

Since $J$ is finite, it suffices to show that, for each $j=1, \ldots, J$,

$$
\sup _{\xi \in \mathcal{M}_{j, \eta}(\delta)}\left|\bar{\nu}_{n}\left(\Xi_{0}+n^{-1 / 2} \xi\right)-\bar{\nu}_{n}\left(\Xi_{0}\right)\right|(w)=o_{p}(1) .
$$

An application of the triangle inequality yields that, for every $\xi \in \mathcal{M}_{j, \eta}(\delta)$,

$$
\begin{aligned}
\left|\bar{\nu}_{n}\left(\Xi_{0}+n^{-1 / 2} \xi\right)-\bar{\nu}_{n}\left(\Xi_{0}\right)\right|(w) \leq & \left|\bar{\nu}_{n}\left(\Xi_{0}+n^{-1 / 2} \xi\right)-\bar{\nu}_{n}\left(\Xi_{0}+n^{-1 / 2} \xi^{(j)}\right)\right|(w) \\
& +\left|\bar{\nu}_{n}\left(\Xi_{0}+n^{-1 / 2} \xi^{(j)}\right)-\bar{\nu}_{n}\left(\Xi_{0}\right)\right|(w) .
\end{aligned}
$$

We separately consider two terms on the right-hand side of the above inequality. First, we can form an envelop function $I_{i, j}^{(1)}(\delta):=1\left\{\max _{1 \leq k \leq K}\left|\tilde{V}_{i}+n^{-1 / 2} \xi^{(j)} \circ \Pi_{\eta}\left(V_{2 i}\right)-w\right| \leq n^{-1 / 2} \delta\right\}$ for a collection of functions

$$
\left\{1\left\{\tilde{V}_{i}+n^{-1 / 2} \xi\left(V_{2 i}\right) \leq w\right\}-1\left\{\tilde{V}_{i}+n^{-1 / 2} \xi^{(j)} \circ \Pi_{\eta}\left(V_{2 i}\right) \leq w\right\}: \xi \in \mathcal{M}_{j, \eta}(\delta)\right\} .
$$


We can write

$$
\sup _{\xi \in \mathcal{M}_{j, \eta}(\delta)}\left|\bar{\nu}_{n}\left(\Xi_{0}+n^{-1 / 2} \xi\right)-\bar{\nu}_{n}\left(\Xi_{0}+n^{-1 / 2} \xi^{(j)}\right)\right|(w) \leq n^{-1 / 2} \sum_{i=1}^{n} I_{i, j}^{(1)}(\delta)+\sqrt{n} E\left[I_{1, j}^{(1)}(\delta)\right] .
$$

Let $\xi_{k}^{(j)}:=\xi^{(j)} \circ \prod_{\eta}\left(v_{k}\right)$ for $k=1, \ldots, K$. The second term on the right-hand of $\mathrm{A} 8$ become arbitrarily small for a sufficiently small $\delta>0$, because we have

$$
\sqrt{n} E\left[I_{1, j}^{(1)}(\delta)\right] \leq \sqrt{n} \sum_{k=1}^{K} \int_{-n^{-1 / 2} \delta}^{n^{-1 / 2} \delta} f_{\tilde{V}}\left(s-\xi_{k}^{(j)}+w\right) d s \leq \delta C_{1},
$$

for some constant $C_{1}$. Applying the Markov inequality for the first term on the right-hand of (A8), we obtain

$$
\operatorname{Pr}\left\{n^{-1 / 2} \sum_{i=1}^{n} I_{i, j}^{(1)}(\delta) \geq \epsilon\right\} \leq \epsilon^{-1} \sqrt{n} E\left[I_{1, j}(\delta)\right],
$$

where the right-hand side becomes arbitrarily small for a sufficiently small $\delta$ due to (A9). Thus, the right-hand side of (A8) converges to 0 in probability for a sufficiently small $\delta>0$.

Next, we have the remaining term. We have, for every $i=1, \ldots, n$,

$$
\left|1\left\{\tilde{V}_{i}+n^{-1 / 2} \xi^{(j)} \circ \Pi_{\eta}\left(V_{2 i}\right) \leq w\right\}-1\left\{\tilde{V}_{i} \leq w\right\}\right| \leq I_{i, j}^{(2)}(\delta)
$$

where $I_{i, j}^{(2)}(\delta):=1\left\{\left|\tilde{V}_{i}-w\right| \leq n^{-1 / 2} \max _{1 \leq k \leq K}\left|\xi_{k}^{(j)}\right|\right\}$. Using the Markov inequality, we can show that

$$
\operatorname{Pr}\left\{\left|\bar{\nu}_{n}\left(\Xi_{0}+n^{-1 / 2} \xi^{(j)}\right)-\bar{\nu}_{n}\left(\Xi_{0}\right)\right|(w) \geq \epsilon\right\} \leq \epsilon^{-2} E\left[I_{i, j}^{(2)}(\delta)\right],
$$

where the right-hand side goes to zero as $n \rightarrow \infty$ because $E\left[I_{i, j}^{(2)}(\delta)\right] \leq C_{2} n^{-1 / 2}$ for some constant $C_{2}$. Hence the proof is completed.

Lemma A4. Let $\phi_{n} \in \ell(\mathcal{W})$ be the function defined in $(A 4)$. Suppose that $\sqrt{n}\left(F_{n}-F_{0}\right)$ converges in distribution to a tight, random element in $\left(\ell^{\infty}(\mathcal{V})\right)^{2}$. Then,

$$
\sqrt{n}\left(\phi_{n}\left(F_{n}\right)-\phi\left(F_{0}\right)\right)=\nu_{n}\left(F_{0}\right)+\phi_{F_{0}}^{\prime}\left(\sqrt{n}\left(F_{n}-F_{0}\right)\right)+o_{p}(1),
$$

uniformly in $\mathcal{W}$, where $\phi_{F_{0}}^{\prime}$ is the Hadamard derivative given in Lemma A2.

Proof. By definition, we can write

$$
\sqrt{n}\left(\phi_{n}\left(F_{n}\right)-\phi\left(F_{0}\right)\right)=\nu_{n}\left(F_{n}\right)+\sqrt{n}\left(\phi\left(F_{n}\right)-\phi\left(F_{0}\right)\right) .
$$

First, Lemma A3 shows that, uniformly in $\mathcal{W}$,

$$
\nu_{n}\left(F_{n}\right)=\nu_{n}\left(F_{0}\right)+o_{p}(1)
$$

Since the map $\phi$ is Hadamard differentiable, the functional delta method in Theorem 3.9.4 of van der Vaart and Wellner (1996) with Lemma A2 implies that

$$
\sqrt{n}\left(\phi\left(F_{n}\right)-\phi\left(F_{0}\right)\right)=\phi_{F_{0}}^{\prime}\left(\sqrt{n}\left(F_{n}-F_{0}\right)\right)+o_{p}(1) .
$$

Hence the desired result follows.

Proof of Lemma 1. The result follows from the functional central limit theorem for empirical distribution functions. See Chapter 2 of van der Vaart and Wellner (1996) for instance. 
Proof of Proposition 1. Let $x \in \mathcal{X}$ be fixed. From its definition in (3), the counterfactual distribution estimator $\hat{F}_{Y_{t}(0) \mid X=x, D_{t}=1}$ can be considered as an empirical distribution indexed by estimated distribution functions $\hat{F}_{Y_{t-1 \mid X=x, D_{t}=0}}$ and $\hat{F}_{Y_{t-1 \mid X=x, D_{t}=1}}$. Thus, there exists some map $\phi_{n}: \mathcal{C}\left(\mathcal{Y}_{t-1 \mid x, 0}\right) \times \mathcal{C}\left(\mathcal{Y}_{t-1 \mid x, 0}\right) \mapsto \mathcal{C}\left(\mathcal{Y}_{t-1 \mid x, 1}(0)\right)$ such that

$$
\hat{F}_{Y_{t}(0) \mid X=x, D_{t}=1}=\phi_{n}\left(\hat{F}_{Y_{t-1 \mid X=x, D_{t}=0}}, \hat{F}_{Y_{t-1 \mid X=x, D_{t}=1}}\right) .
$$

The empirical processes of $\hat{F}_{Y_{t-1 \mid X=x, D_{t}=0}}$ and $\hat{F}_{Y_{t-1 \mid X=x, D_{t}=1}}$ are given by $\hat{G}_{t-1, x}^{(0)}$ and $\hat{G}_{t-1, x}^{(1)}$, respectively, and Lemma 1 shows that they jointly have a tight limit asymptotically. Also, notice that $\phi_{n}\left(F_{Y_{t-1 \mid X=x, D_{t}=0}}, F_{Y_{t-1 \mid X=x, D_{t}=1}}\right)=\tilde{F}_{Y_{t}(0) \mid X=x, D_{t}=1}$ and that the empirical process $\tilde{F}_{Y_{t}(0) \mid X=x, D_{t}=1}$ is $\tilde{G}_{t, x}^{(0)}$ as defined in $(4)$. It follows from Lemma A2 and A4 that

$$
\sqrt{n}\left(\hat{F}_{Y_{t}(0) \mid X=x, D_{t}=1}-F_{Y_{t}(0) \mid X=x, D_{t}=1}\right)=r_{x}^{(0)} \tilde{G}_{t, x}^{(0)}+\kappa_{x}\left(\hat{G}_{t-1, x}^{(0)}, \hat{G}_{t-1, x}^{(1)}\right)+o_{p}(1),
$$

uniformly in $\mathcal{Y}_{t \mid x, 1}(0)$, where $\kappa_{x}$ represents the Hadamard derivative of $\phi_{n}$ as shown in Lemma A2. Hence the extended continuous mapping theorem with Lemma 1 yields the desired result.

Proof of Theorem 2. Let $x \in \mathcal{X}$ be fixed. When $\hat{F}_{Y_{t}(j) \mid X=x, D_{t}=1}(y)$ is weakly increasing in $y$, we can show that the corresponding quantile function $\hat{F}_{Y_{t}(j) \mid X=x, D_{t}=1}^{-1}(\tau)$ is Hadamard differentiable. It follows from the functional delta method that

$$
\sqrt{n}\left(\hat{F}_{Y_{t}(j) \mid X=x, D_{t}=1}^{-1}(\tau)-F_{Y_{t}(j) \mid X=x, D_{t}=1}^{-1}(\tau)\right) \rightsquigarrow\left(\frac{\mathbb{Z}_{x}^{(j)}}{f_{Y_{t}(j) \mid X=x, D_{t}=1}}\right) \circ F_{Y_{t}(j) \mid X=x, D_{t}=1}^{-1}(\tau),
$$

as a stochastic process indexed by $\tau \in \mathcal{T}$ and $j \in\{0,1\}$. Hence the desired result holds.

Proof of Corollary 2. The result follows from the continuous mapping theorem. See, for example, Section 2.1 of Kosorok (2007).

We now prove a technical lemma, which is a bootstrap version of Lemma A4. We respectively denote the bootstrap counterpart of $\phi_{n}$ and $F_{n}$ by $\phi_{n}^{*}$ and $F_{n}^{*}$, which are obtained through bootstrap with some random weights $\left(W_{1}, \ldots, W_{n}\right)$ satisfying Assumption B.

Lemma A5. Suppose that both $\sqrt{n}\left(F_{n}-F_{0}\right)$ and $\sqrt{n}\left(F_{n}^{*}-F_{0}\right)$ converge in distribution to some tight random elements unconditional on the original sample. Then, uniformly in $\mathcal{W}$,

$$
\sqrt{n}\left(\phi_{n}^{*}\left(F_{n}^{*}\right)-\phi_{n}\left(F_{n}\right)\right)=\sqrt{n}\left(\phi_{n}^{*}\left(F_{0}\right)-\phi_{n}\left(F_{0}\right)\right)+\phi_{F_{0}}^{\prime}\left(\sqrt{n}\left(F_{n}^{*}-F_{n}\right)\right)+o_{p}(1) .
$$

Proof. Let $\tilde{\nu}_{n}^{*}(F):=\sqrt{n}\left(\phi_{n}^{*}(F)-\phi(F)\right)$ for $F \in\left(\ell^{\infty}(\mathcal{V})\right)^{2}$. We have

$$
\sqrt{n}\left(\phi_{n}^{*}\left(F_{n}^{*}\right)-\phi\left(F_{0}\right)\right)=\tilde{\nu}_{n}^{*}\left(F_{n}\right)+\sqrt{n}\left(\phi\left(F_{n}^{*}\right)-\phi\left(F_{0}\right)\right) .
$$

By a similar argument used to prove Lemma A3, we can show that, uniformly in $\mathcal{W}$,

$$
\tilde{\nu}_{n}^{*}\left(F_{n}\right)=\tilde{\nu}_{n}^{*}\left(F_{0}\right)+o_{p}(1) \text {. }
$$


Also $\phi$ is the Hadamard differentiable function $\phi$ and $\sqrt{n}\left(F_{n}^{*}-F_{0}\right)$ converges in distribution to a tight random element unconditional on the original sample. Thus the functional delta method implies that, uniformly in $\mathcal{W}$,

$$
\sqrt{n}\left(\phi\left(F_{n}^{*}\right)-\phi\left(F_{0}\right)\right)=\phi_{F_{0}}^{\prime}\left(\sqrt{n}\left(F_{n}^{*}-F_{0}\right)\right)+o_{p}(1) .
$$

It follows from A10 and A11 that, uniformly in $\mathcal{W}$,

$$
\sqrt{n}\left(\phi_{n}^{*}\left(F_{n}^{*}\right)-\phi\left(F_{0}\right)\right)=\tilde{\nu}_{n}^{*}\left(F_{0}\right)+\phi_{F_{0}}^{\prime}\left(\sqrt{n}\left(F_{n}^{*}-F_{0}\right)\right)+o_{p}(1),
$$

which together with Lemma A4 yields the desired result because $\phi_{F_{0}}^{\prime}$ is a linear map.

Proof of Lemma 2. The result follows from Theorem 3.6.13 of van der Vaart and Wellner (1996). Thus we omit the detail.

Proof of Theorem 3. Let $x \in \mathcal{X}$ be fixed. First we wish to show that $\hat{Z}_{x}^{*} \rightsquigarrow \mathbb{Z}_{x}$, where $\hat{Z}_{x}^{*}:=\left(\hat{Z}_{x}^{(0) *}, \hat{Z}_{x}^{(1) *}\right)^{\prime}$ and $\mathbb{Z}_{x}:=\left(\mathbb{Z}_{x}^{(0)}, \mathbb{Z}_{x}^{(1)}\right)^{\prime}$. Define $\tilde{Z}_{x}^{*}:=\left(\tilde{Z}_{x}^{(0) *}, \hat{Z}_{x}^{(1) *}\right)^{\prime}$, where $\tilde{Z}_{x}^{(0) *}:=$ $r_{x}^{(0)} \tilde{G}_{t, x}^{(0) *}+\kappa_{x}\left(\hat{G}_{t-1, x}^{*}, \hat{G}_{t, x}^{*}\right)$, with $\tilde{G}_{t, x}^{(0) *}, \hat{G}_{t-1, x}^{*}$ and $\hat{G}_{t, x}^{*}$ denoting the bootstrap version of the empirical processes $\tilde{G}_{t, x}^{(0)}, \hat{G}_{t-1, x}$ and $\hat{G}_{t, x}$, respectively. By the triangle inequality, we obtain

$$
\begin{aligned}
\sup _{h \in B L_{1}}\left|E_{M}\left[h\left(\hat{Z}_{x}^{*}\right)\right]-E\left[h\left(\mathbb{Z}_{x}\right)\right]\right| \leq & \sup _{h \in B L_{1}}\left|E_{M}\left[h\left(\hat{Z}_{x}^{*}\right)\right]-E_{M}\left[h\left(\tilde{Z}_{x}^{*}\right)\right]\right| \\
& +\sup _{h \in B L_{1}}\left|E_{M}\left[h\left(\tilde{Z}_{x}^{*}\right)\right]-E\left[h\left(\mathbb{Z}_{x}\right)\right]\right| .
\end{aligned}
$$

It suffices to show that $\mathrm{A} 12$ and $\mathrm{A} 13$ converge in probability to zero, separately.

We consider $\left[\right.$ A12). Because $\left|E_{M}\left[h\left(\hat{Z}_{x}^{*}\right)\right]-E_{M}\left[h\left(\tilde{Z}_{x}^{*}\right)\right]\right| \leq E_{M}\left|h\left(\hat{Z}_{x}^{*}\right)-h\left(\tilde{Z}_{x}^{*}\right)\right|$, we have

$$
E\left[\sup _{h \in B L_{1}}\left|E_{M}\left[h\left(\hat{Z}_{x}^{*}\right)\right]-E_{M}\left[h\left(\tilde{Z}_{x}^{*}\right)\right]\right|\right] \leq E\left[\sup _{h \in B L_{1}}\left|h\left(\hat{Z}_{x}^{*}\right)-h\left(\tilde{Z}_{x}^{*}\right)\right|\right] .
$$

Let $\epsilon>0$ be fixed and define $I_{n, \epsilon}^{*}:=1\left\{\left\|\hat{Z}_{x}^{*}-\tilde{Z}_{x}^{*}\right\|_{\infty}>\epsilon\right\}$. Lemma 2 and A5 imply that $\lim _{n \rightarrow \infty} E\left[I_{n, \epsilon}^{*}\right] \leq \epsilon$, while $\sup _{h \in B L_{1}}\left|h\left(\hat{Z}_{x}^{*}\right)-h\left(\tilde{Z}_{x}^{*}\right)\right| \leq 2$. It follows that

$$
E\left[\sup _{h \in B L_{1}}\left|h\left(\hat{Z}_{x}^{*}\right)-h\left(\tilde{Z}_{x}^{*}\right)\right| \cdot I_{n, \epsilon}^{*}\right] \leq 2 \epsilon
$$

Also we can show that

$$
E\left[\sup _{h \in B L_{1}}\left|h\left(\hat{Z}_{x}^{*}\right)-h\left(\tilde{Z}_{x}^{*}\right)\right| \cdot\left(1-I_{n, \epsilon}^{*}\right)\right] \leq \epsilon
$$

because $\sup _{h \in B L_{1}}\left|h\left(\hat{Z}_{x}^{*}\right)-h\left(\tilde{Z}_{x}^{*}\right)\right| \leq\left\|\hat{Z}_{x}^{*}(y)-\tilde{Z}_{x}^{*}(y)\right\|_{\infty}$. It follows from A15 and A16) that the right-hand side of A14 is bounded by $3 \epsilon$. Since $\epsilon$ is arbitrary, an application of the Markov inequality yields the convergence of (A12) to 0 in probability.

Consider (A13). Using Lemma 2 together with the continuous mapping theorem, we can show that (A13) converges to 0 in probability. Hence we obtain the desired result.

We now consider validity of exchangeable bootstrap for the CQTT. Theorem 3.9.11 of van der Vaart and Wellner (1996) shows that the functional delta method can apply for Hadamard differentiable maps under resampling. Since the map from distribution to quantile is Hadamard differentiable, the desired result follows. 


\section{Tables and Figures}

Table 1: Monte Carlo Simulations, DGP 1

\begin{tabular}{lccccccc}
\hline & & \multicolumn{3}{c}{ DDID } & \multicolumn{3}{c}{ CIC } \\
& $N$ & 0.1 & 0.5 & 0.9 & 0.1 & 0.5 & 0.9 \\
\hline TE=0 & & & & & & & \\
Bias & 100 & 0.044 & 0.045 & 0.081 & 0.012 & -0.097 & -0.295 \\
& 200 & 0.016 & 0.021 & 0.066 & -0.009 & -0.048 & -0.141 \\
& 500 & 0.016 & 0.008 & 0.023 & -0.005 & -0.042 & -0.074 \\
Rej. Prob. & 100 & 0.042 & 0.037 & 0.023 & 0.042 & 0.041 & 0.100 \\
& 200 & 0.049 & 0.050 & 0.044 & 0.051 & 0.056 & 0.076 \\
& 500 & 0.043 & 0.047 & 0.034 & 0.035 & 0.043 & 0.069 \\
\hline TE=1 & & & & & & & \\
Bias & 100 & 0.059 & 0.064 & 0.109 & 0.251 & -0.051 & -0.293 \\
& 200 & 0.031 & 0.027 & 0.049 & 0.128 & -0.052 & -0.200 \\
& 500 & 0.014 & 0.019 & 0.025 & 0.053 & -0.019 & -0.090 \\
Rej. Prob. & 100 & 0.397 & 0.675 & 0.359 & 0.409 & 0.617 & 0.548 \\
& 200 & 0.742 & 0.949 & 0.703 & 0.713 & 0.859 & 0.614 \\
& 500 & 0.994 & 1.000 & 0.992 & 0.983 & 0.992 & 0.756 \\
\hline
\end{tabular}

Notes: Each Monte Carlo simulation uses 1000 bootstrap iterations. Each cell lists the bias and the rejection probabilities from 1000 Monte Carlo simulations. To calculate empirical rejection probabilities, we set the nominal size to be $5 \%$. 
Table 2: Monte Carlo Simulations, DGP 2

\begin{tabular}{ccccccc}
\hline \multicolumn{3}{c}{$\mathbf{T E}=\mathbf{0}$} & \multicolumn{3}{c}{$\mathbf{T E}=\mathbf{1}$} \\
$\bar{\rho}$ & 0.1 & 0.5 & 0.9 & 0.1 & 0.5 & 0.9 \\
\hline Bias & & & & & & \\
0.00 & 0.020 & 0.034 & 0.037 & 0.023 & 0.023 & 0.050 \\
0.05 & 0.073 & 0.023 & 0.012 & 0.088 & 0.029 & 0.008 \\
0.10 & 0.121 & 0.028 & -0.033 & 0.112 & 0.019 & -0.032 \\
0.50 & 0.425 & 0.013 & -0.374 & 0.435 & 0.027 & -0.353 \\
RMSE & & & & & & \\
0.00 & 0.348 & 0.261 & 0.340 & 0.342 & 0.248 & 0.359 \\
0.05 & 0.348 & 0.256 & 0.324 & 0.358 & 0.258 & 0.342 \\
0.10 & 0.374 & 0.260 & 0.352 & 0.374 & 0.259 & 0.346 \\
0.50 & 0.565 & 0.272 & 0.529 & 0.566 & 0.264 & 0.508 \\
\hline
\end{tabular}

Notes: Each Monte Carlo simulation uses 1000 bootstrap iterations. Each cell lists the bias and the root mean squared error from 1000 Monte Carlo simulations. Here, $\bar{\rho}$ controls whether or not the the Copula Invariance assumption is violated. When $\bar{\rho}=0$, the Copula Invariance assumption holds; the further away $\bar{\rho}$ is from 0 , the more strongly the Copula Invariance assumption is violated. 
Table 3: Summary Statistics (averages)

\begin{tabular}{lccc}
\hline & Treated States & Untreated States & Difference (p-value) \\
\hline White & 0.89 & 0.88 & $0.014(0.06)$ \\
Male & 0.48 & 0.49 & $-0.008(0.52)$ \\
College Degree & 0.45 & 0.41 & $0.039(0.00)$ \\
Log Earnings & 6.39 & 6.33 & $0.065(0.00)$ \\
\hline
\end{tabular}

Notes: The second and third columns report sample averages for states that raised their minimum wage during the first quarter of 2007 (treated states) and states that had their minimum wage equal to the federal minimum wage for the entire period. The last column presents differences between the figures in the second and third columns with $p$-values in parentheses.

Sources: Panel data from the Current Population Survey (CPS) (Flood et al., 2015). 
Table 4: Conditional QTT Estimates

\begin{tabular}{|c|c|c|c|c|c|c|c|}
\hline \multicolumn{3}{|c|}{ Subgroup } & \multirow[b]{2}{*}{$N$} & \multirow[b]{2}{*}{ Reject $H_{0}$} & \multicolumn{3}{|c|}{ Quantile } \\
\hline Race & Gender & Education & & & 0.1 & 0.5 & 0.9 \\
\hline \multirow[t]{8}{*}{ White } & \multirow[t]{4}{*}{ Male } & College & \multirow[t]{2}{*}{1617} & & 0.004 & 0.007 & 0.000 \\
\hline & & & & & $(0.048)$ & $(0.031)$ & $(0.037)$ \\
\hline & & \multirow[t]{2}{*}{ Non-College } & \multirow[t]{2}{*}{2306} & & -0.021 & -0.019 & 0.075 \\
\hline & & & & & $(0.068)$ & $(0.028)$ & $(0.068)$ \\
\hline & \multirow[t]{4}{*}{ Female } & College & 1629 & Yes & -0.029 & 0.027 & -0.046 \\
\hline & & & & & $(0.056)$ & $(0.033)$ & $(0.052)$ \\
\hline & & Non-College & 1980 & & -0.038 & -0.043 & -0.086 \\
\hline & & & & & $(0.054)$ & $(0.035)$ & $(0.068)$ \\
\hline \multirow[t]{8}{*}{ Non-White } & \multirow[t]{4}{*}{ Male } & College & 156 & & -0.492 & -0.07 & 0.292 \\
\hline & & & & & $(0.265)$ & $(0.161)$ & $(0.268)$ \\
\hline & & Non-College & 282 & Yes & -0.087 & -0.044 & 0.036 \\
\hline & & & & & $(0.186)$ & $(0.095)$ & $(0.136)$ \\
\hline & \multirow[t]{4}{*}{ Female } & College & 209 & & 0.097 & 0.033 & 0.007 \\
\hline & & & & & $(0.172)$ & $(0.088)$ & $(0.121)$ \\
\hline & & Non-College & 340 & Yes & -0.25 & -0.026 & -0.043 \\
\hline & & & & & $(0.175)$ & $(0.086)$ & $(0.162)$ \\
\hline
\end{tabular}

Notes: Conditional QTTs by race, gender, and education subgroups. $N$ is the number of observations in each group. The column of "Reject $H_{0}$ " reports whether the null of no effect at any quantile is rejected using the Kolmogorov-Smirnov test with nominal size of $5 \%$ using equally spaced quantiles from 0.05 to 0.95 by 0.01 and is based on 1000 bootstrap iterations to calculate critical values. The last three columns report conditional QTTs at the $0.1,0.5$, and 0.9 quantiles. Standard errors are pointwise and computed using the bootstrap with 1000 iterations. Sources: Panel data from the Current Population Survey (CPS) (Flood et al., 2015) 
Figure 1: Conditional QTTs of Minimum Wage Increase on Earnings (with 95\% Confidence Bands)
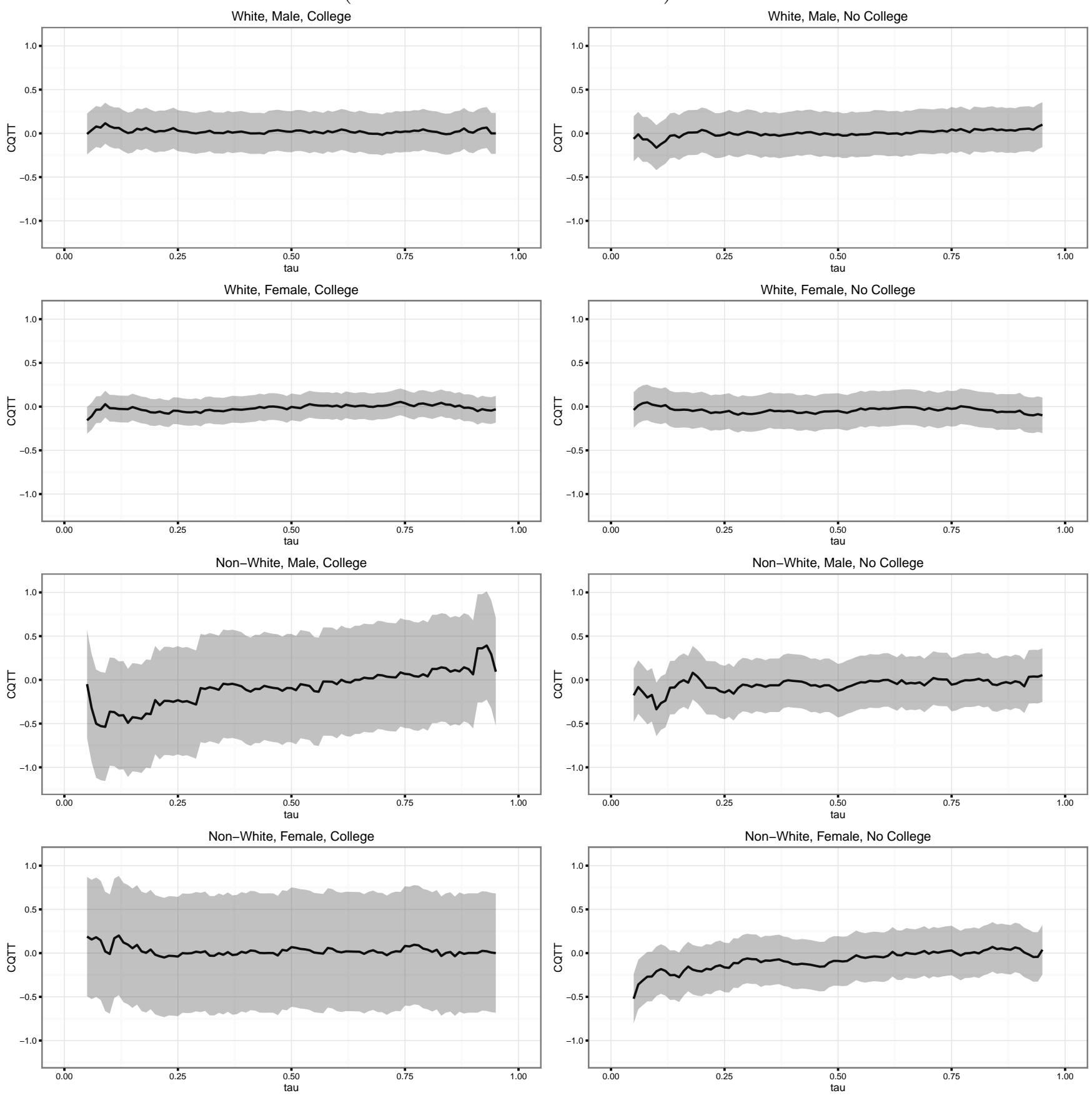

Notes: Conditional QTT estimates for groups formed by race, gender, and education. The figure also provides $95 \%$ confidence bands. These are formed by inverting KolmogorovSmirnov statistics and based on 1000 bootstrap iterations. Sources: Panel data from the Current Population Survey (CPS) (Flood et al., 2015). 